Proceedings of the Edinburgh Mathematical Society (2006) 49, 215-239 (C)

DOI:10.1017/S0013091504001610 Printed in the United Kingdom

\title{
ESTIMATES OF BLOW-UP TIME FOR A NON-LOCAL REACTIVE-CONVECTIVE PROBLEM MODELLING OHMIC HEATING OF FOODS
}

\author{
C. V. NIKOLOPOULOS ${ }^{1}$ AND D. E. TZANETIS ${ }^{2}$ \\ ${ }^{1}$ Department of Mathematics, University of the Aegean, \\ 83200 Samos, Greece (cnikolo@aegean.gr) \\ ${ }^{2}$ Department of Mathematics, School of Applied Mathematical and \\ Physical Sciences, National Technical University of Athens, \\ Zografou Campus, 15780 Athens, Greece (dtzan@math.ntua.gr)
}

(Received 23 December 2004)

\begin{abstract}
In this work, we estimate the blow-up time for the non-local hyperbolic equation of ohmic type, $u_{t}+u_{x}=\lambda f(u) /\left(\int_{0}^{1} f(u) \mathrm{d} x\right)^{2}$, together with initial and boundary conditions. It is known that, for $f(s),-f^{\prime}(s)$ positive and $\int_{0}^{\infty} f(s) \mathrm{d} s<\infty$, there exists a critical value of the parameter $\lambda>0$, say $\lambda^{*}$, such that for $\lambda>\lambda^{*}$ there is no stationary solution and the solution $u(x, t)$ blows up globally in finite time $t^{*}$, while for $\lambda \leqslant \lambda^{*}$ there exist stationary solutions. Moreover, the solution $u(x, t)$ also blows up for large enough initial data and $\lambda \leqslant \lambda^{*}$. Thus, estimates for $t^{*}$ were found either for $\lambda$ greater than the critical value $\lambda^{*}$ and fixed initial data $u_{0}(x) \geqslant 0$, or for $u_{0}(x)$ greater than the greatest steadystate solution (denoted by $w_{2} \geqslant w^{*}$ ) and fixed $\lambda \leqslant \lambda^{*}$. The estimates are obtained by comparison, by asymptotic and by numerical methods. Finally, amongst the other results, for given $\lambda, \lambda^{*}$ and $0<$ $\lambda-\lambda^{*} \ll 1$, estimates of the following form were found: upper bound $\epsilon+c_{1} \ln \left[c_{2}\left(\lambda-\lambda^{*}\right)^{-1}\right]$; lower bound $c_{3}\left(\lambda-\lambda^{*}\right)^{-1 / 2}$; asymptotic estimate $t^{*} \sim c_{4}\left(\lambda-\lambda^{*}\right)^{-1 / 2}$ for $f(s)=\mathrm{e}^{-s}$. Moreover, for $0<\lambda \leqslant \lambda^{*}$ and given initial data $u_{0}(x)$ greater than the greatest steady-state solution $w_{2}(x)$, we have upper estimates: either $c_{5} \ln \left(c_{6} A_{0}^{-1}+1\right)$ or $\epsilon+c_{7} \ln \left(c_{8} \zeta^{-1}\right)$, where $A_{0}, \zeta$ measure, in some sense, the difference $u_{0}-w_{2}$ (if $u_{0} \rightarrow w_{2}+$, then $A_{0}, \zeta \rightarrow 0+$ ). $c_{i}>0$ are some constants and $0<\epsilon \ll 1,0<A_{0}, \zeta$. Some numerical results are also given.
\end{abstract}

Keywords: non-local hyperbolic equations; blow-up; estimates of blow-up time; asymptotic and numerical estimates

2000 Mathematics subject classification: Primary 35L60

Secondary 35Q80

\section{Introduction}

We consider the non-local initial boundary-value problem

$$
\begin{gathered}
u_{t}(x, t)+u_{x}(x, t)=\lambda \frac{f(u(x, t))}{\left(\int_{0}^{1} f(u(x, t)) \mathrm{d} x\right)^{2}}, \quad 0<x<1, \quad t>0, \\
u(0, t)=0, \quad t>0, \\
u(x, 0)=u_{0}(x) \geqslant 0, \quad 0<x<1,
\end{gathered}
$$


where $\lambda>0$. The function $u(x, t)$ represents the dimensionless temperature when an electric current flows through a conductor (e.g. food) with temperature dependent on electrical resistivity $f(u)>0$, subject to a fixed potential difference $V>0$. The (dimensionless) resistivity $f(u)$ may be either an increasing or a decreasing function of temperature depending strongly on the type of material (food). Problem (1.1) models one of the main methods for sterilizing food. The sterilization can take place by rapidly heating the food electrically. The food is passed through a conduit, part of which lies between two electrodes. A high electric current flowing between the electrodes results in ohmic heating of the food, which quickly gets hot. The problem was considered by Please et al. [24], who looked at the stability of models allowing for different types of flow. More background on this type of process can be found in [5], [12], [15], [26], [27] and $[\mathbf{2 9}]$.

Lacey et al. [22] have also studied problem (1.1): for $f$ decreasing with $\int_{0}^{\infty} f(s) \mathrm{d} s<\infty$, blow-up occurs if $\lambda$ is too large for a steady state to exist or if the initial condition is too big. If $f$ is increasing with $\int_{0}^{\infty} \mathrm{d} s / f(s)<\infty$, blow-up is also possible. If $f$ is increasing with $\int_{0}^{\infty} \mathrm{d} s / f(s)=\infty$ or decreasing with $\int_{0}^{\infty} f(s) \mathrm{d} s=\infty$, the solution is global in time; some special cases with particular forms of $f$ are discussed to illustrate what the solution can do (for details see $[\mathbf{2 2}]$ and the references therein).

Here $\lambda$ is a dimensionless parameter and can be equal to (amongst other things) the square of (its relationship is actually proportional to) the applied potential difference, $V$. In the case where $f(u)$ is a sufficiently rapidly decreasing function of temperature, there exists a critical value of the potential difference $V$, say $V^{*}$, such that, for $V>V^{*}$ (equivalently, $\lambda>\lambda^{*}$ ), a thermal runaway (blow-up of the temperature $u$ or burning of the food) takes place (see $[\mathbf{1 9}, \mathbf{2 0}, \mathbf{2 2}]$ ).

Below we assume that $f$ satisfies

$$
\begin{gathered}
f(s)>0, \quad f^{\prime}(s)<0, \quad s \geqslant 0, \\
\int_{0}^{\infty} f(s) \mathrm{d} s<\infty
\end{gathered}
$$

for instance, either $f(s)=\mathrm{e}^{-s}$ or $f(s)=(1+s)^{-p}, p>1$, satisfy (1.2).

For the initial data it is required that $u_{0}(x), u_{0}^{\prime}(x)$ be bounded, $u_{0}(x) \geqslant 0$ in $[0,1]$ (the latter requirement is a consequence of the fact that for any initial data the solution $u$ becomes non-negative over $(0,1]$ for some time $t$ and so, with an appropriate redefinition of $t$, we can always make this assumption $[\mathbf{1 9}, \mathbf{2 2}])$.

The solution $u(x, t)$ also blows up for large enough initial data even if $0<\lambda \leqslant \lambda^{*}$ $[\mathbf{1 9}, \mathbf{2 0}, \mathbf{2 2}]$; in this case, we give (see $\S 5$ ) an analogous estimate of blow-up time, as for the case of $\lambda>\lambda^{*}$.

The steady-state problem that corresponds to (1.1) is

$$
w^{\prime}=\mu f(w)=\lambda \frac{f(w)}{\left(\int_{0}^{1} f(w) \mathrm{d} x\right)^{2}}, \quad 0<x<1, \quad w(0)=0,
$$

where $w=w(x)=w(x ; \lambda)($ see $[\mathbf{6}, \mathbf{1 4}, \mathbf{1 9}, \mathbf{2 0}, \mathbf{2 2}])$. The parameter $\mu$ is referred to as a local parameter, while $\lambda$ is referred to as a non-local one and the relation between them is $\mu=\lambda /\left(\int_{0}^{1} f(w) \mathrm{d} x\right)^{2}$. 

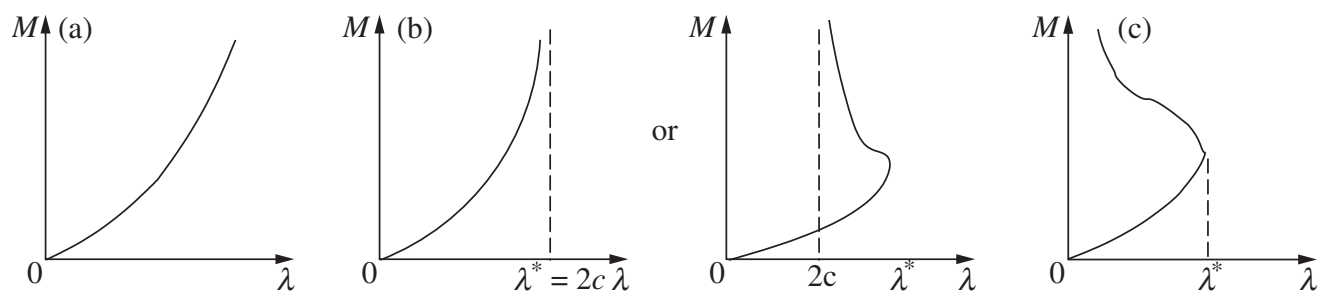

Figure 1. Possible response diagrams for equilibrium solutions: (a) $M f(M)$ is unbounded; (b) $M f(M) \rightarrow c, 0<c<\infty$, as $M \rightarrow \infty$; (c) $M f(M) \rightarrow 0$, as $M \rightarrow \infty$. In each part of the figure it is possible for there to be more turning points than are shown (so that for some $\lambda$ there are more solutions).

On integrating the ordinary differential equation (1.3) we see that $\lambda=M^{2} / \mu$, where $M=M(\mu)=\max _{[0,1]} w=w(1 ; \mu)$ and $\mu=\int_{0}^{M} \mathrm{~d} s / f(s)$.

It is clear that $M(\mu) \rightarrow 0+$ as $\mu \rightarrow 0+$ and, with $f(0)>0, \lambda \rightarrow 0+$ as $\mu \rightarrow 0+$.

It is also known that if $(1.2 b)$ holds, then there exists a critical value of the parameter $\lambda$, say $\lambda^{*}<\infty$, such that, for $\lambda>\lambda^{*}$, the solution $u(x, t ; \lambda)$ to problem (1.1) blows up globally in finite time $t^{*}\left(u \rightarrow \infty\right.$ for all $x \in(0,1]$ as $t \rightarrow t^{*}-$; actually, the blowup is uniform in $x$ on compact subsets of $(0,1]$ : for two points $0<x_{1}<x_{2} \leqslant 1$, $\left|u\left(x_{1}, t\right)-u\left(x_{2}, t\right)\right| \rightarrow 0$ as $t \rightarrow t^{*}[\mathbf{2 2}]$ ) and problem (1.3) has no solutions (of any kind). For a fixed $\lambda \in\left(0, \lambda^{*}\right)$ there exist at least two solutions $w(x ; \lambda)$ and a unique $u(x, t ; \lambda) ; u(x, t ; \lambda)$ may either exist for all time or blow up globally depending on the initial data $[\mathbf{1 9}, \mathbf{2 0}, \mathbf{2 2}]$ (for the blow-up, $u_{0}$ must be greater than the greatest steady-state solution $w(x ; \lambda)$ and (1.2) holds). The response (bifurcation) diagrams for problem (1.3) are as in Figure 1 (see also $[\mathbf{2 2}]$ ).

Our purpose, in this work, is to find some estimates of the blow-up time $t^{*}$, either with respect to the parameter $\lambda$ (more precisely, with respect to the difference $\lambda-\lambda^{*}$ ), when $\lambda>\lambda^{*}$ and with fixed initial data $u_{0}(x)$, or with respect to initial data $u_{0}(x)$ and with fixed $\lambda \leqslant \lambda^{*}$.

Work related to this model (the thermistor problem) can be found in $[\mathbf{1}, \mathbf{7 - 1 0}, \mathbf{1 3}]$.

Estimates of blow-up time are very important since they answer the question of 'when' the blow-up takes place $[\mathbf{3}, \mathbf{1 6}, \mathbf{1 7}]$ (for problem (1.1) this is the time when the food is burnt).

In part $(b)$ or part $(c)$ of Figure 1, with $2 c<\lambda^{*}$, there may be either only one or more than one turning point $\left(\lambda^{*}, M^{*}\right)$ depending on $f$. One can find other forms of non-local diagrams in $[\mathbf{1 9}, \mathbf{2 0}, \mathbf{2 2}]$; their shapes depend strongly on the boundary conditions and the function $f$.

Under the assumptions (1.2), problem (1.3) has at least one classical (regular) steadystate solution $w^{*}=w\left(x ; \lambda^{*}\right)$ (more than one $w^{*}$ may exist). We assume below that $w^{*}$ is unique, i.e. as in Figure $1 c$, and that the pair $(\underline{w}, \bar{w})$ at $\lambda<\lambda^{*}\left(\lambda\right.$ close to $\left.\lambda^{*}\right)$ has the following property: $\underline{w}=w_{1}$ is stable while $\bar{w}=w_{2}$ is unstable (since, in our proofs, we require only the existence of at least one $w^{*}$ at $\lambda^{*}$ and that $\underline{w}(x)<\bar{w}(x)$ for $x$ in $(0,1]$, where $\bar{w}$ is the next steady-state solution greater than $\underline{w}(x)$ at $\left.\lambda<\lambda^{*}\right)$. 
We also emphasize that for $\lambda>\lambda^{*}$ and for all $x \in(0,1]$ we have

$$
\begin{array}{r}
F(u)=\frac{f(u)}{\left(\int_{0}^{1} f(u) \mathrm{d} x\right)^{2}} \rightarrow \infty \quad \text { as } t \rightarrow t^{*}-<\infty, \\
u(x, t ; \lambda) \rightarrow \infty \quad \text { as } t \rightarrow t^{*}-<\infty,
\end{array}
$$

the latter means that $u(x, t ; \lambda)$ blows up globally (see $[\mathbf{1 9}, \mathbf{2 0}, \mathbf{2 2}]$ ).

Similar situations pertaining to the blow-up can be found in the study of the (local) reaction-diffusion problem $[\mathbf{2}, \mathbf{1 8}, \mathbf{2 1}]$

$$
\begin{aligned}
u_{t} & =\Delta u+\lambda f(u), & & x \in \Omega, \quad t>0, \\
B(u) & =0, & & x \in \partial \Omega, \quad t>0, \\
u(x, 0) & =u_{0}(x), & & x \in \Omega,
\end{aligned}
$$

where $B$ represents Dirichlet or Robin boundary conditions, $\Omega$ is a bounded domain of $\mathbb{R}^{n}, \lambda$ is a positive parameter, and $f(u)$ behaves like $\mathrm{e}^{u}$ :

$$
f(s)>0, \quad f^{\prime}(s)>0, \quad f^{\prime \prime}(s) \geqslant 0, \quad \text { for } s \geqslant 0 \quad \text { and } \quad \int_{0}^{\infty} \frac{\mathrm{d} s}{f(s)}<\infty .
$$

Again, under certain conditions, the solution $u$ to (1.5) blows up:

$$
\limsup _{t \rightarrow t^{*}-}\|u(\cdot, t ; \lambda)\|_{\infty}=\infty \text { for } \lambda>\lambda^{*}, t^{*}<\infty .
$$

It should be emphasized that the blow-up for problem (1.5) differs from that of non-local problem (1.1) in that (1.5) does not normally blow up globally. Moreover, there exists a turning point $P^{*}=\left(\lambda^{*},\left\|w^{*}\right\|_{\infty}\right)$ with $\left\|w^{*}\right\|_{\infty}<\infty$ of the response diagram of the steady-state problem corresponding to (1.5). For $f$ satisfying (1.6), the following upper and lower bounds for $t^{*}$ hold: $t_{1} \leqslant t^{*} \leqslant t_{2}$, where $t_{i}=c_{i}\left(\lambda-\lambda^{*}\right)^{-1 / 2}$ and $c_{i}$ are some constants $\left(c_{1}<c_{2}\right)$; also, for $f(s)=\mathrm{e}^{s}, t^{*} \sim K\left(\lambda-\lambda^{*}\right)^{-1 / 2}$ as $\lambda \rightarrow \lambda^{*}+$ asymptotically, where $K$ is a constant [18].

Estimates of blow-up time (upper and lower bounds for $t^{*}$, similar to those for the local problem (1.5)), obtained by using comparison methods, for the non-local equation $u_{t}=u_{x x}+\lambda f(u) /\left(\int_{-1}^{1} f(u) \mathrm{d} x\right)^{2}$, with initial conditions $u_{0}(x)$, Robin-type boundary conditions, decreasing $f$ and $\lambda>\lambda^{*}$, can also be found in [17]. Also, for $f(u)=\mathrm{e}^{-u}$, the following asymptotic estimate holds: $t^{*} \sim t_{u}\left(\lambda-\lambda^{*}\right)^{-1 / 2}$ for $0<\lambda-\lambda^{*} \ll 1$, where $t_{u}$ is a constant. A numerical estimate is also obtained by using a Crank-Nicolson scheme.

In the present work, we find estimates for the non-local problem (1.1), for $f(s)=\mathrm{e}^{-s}$ and for general $f(s)$ satisfying (1.2).

In both problems, estimates of $t^{*}$ have been found only if the spectrum of the steady problem is an interval that is closed on the right, i.e. $\left(0, \lambda^{*}\right]$. It is still an open question how to estimate $t^{*}$ when the spectrum is an open interval $\left(0, \lambda^{*}\right)$ (see $\left.[\mathbf{1 7}, \mathbf{1 8}, \mathbf{2 1}]\right)$. Concerning problem (1.5), there is an upper estimate of blow-up time, given by Bellout [4], which is valid even if the spectrum is open, i.e. $\left(0, \lambda^{*}\right)$; actually, he uses a different method to obtain this estimate and requires $f / f^{\prime}$ to be concave. 
We organize this work as follows: in $\S 2$ we use comparison techniques and find upper and lower bounds for $t^{*}$, when $f$ satisfies (1.2). In $\S 3$ we use an asymptotic expansion and again obtain an estimate of $t^{*}$ but for $f(s)=\mathrm{e}^{-s}$. In $\S 4$ we compute the blow-up time $t^{*}$ numerically by using an upwind scheme and verify the previous estimate. Finally, in $\S 5$ we give an estimate for $t^{*}$ with respect to the initial data $u_{0}(x)$.

\section{Comparison methods: upper and lower bounds of $t^{*}$ for $\lambda>\lambda^{*}$}

If the function $f$ satisfies $(1.2 a)$, one can prove (see the appendix in $[\mathbf{2 2}]$ ) that a maximum principle holds for (1.1) (here is where we need $f$ to be decreasing). We may then, in the usual way, define upper and lower solutions of (1.1): an upper (lower) solution $\bar{u}(\underline{u})$ is defined as a function which satisfies (1.1) if we substitute ' $\geqslant$ ' (' $\leqslant$ ') for '=' (see $[\mathbf{1 9}, \mathbf{2 0}, \mathbf{2 2}, \mathbf{2 5}, \mathbf{2 8}])$. The same comparison properties also hold for the equation $u_{t}+u_{x}=0$ together with the data $(1.1 b),(1.1 c)$. Below we use ideas and techniques similar to those in [17].

\subsection{An upper bound for $t^{*}$}

We now wish to find an upper bound for the blow-up time $t^{*}$. For simplicity, we assume that $0 \leqslant u_{0}<w^{*}$. First, we write (1.3) in a slightly different way,

$$
w^{\prime}=\mu f(w)=\frac{\lambda f(w)}{\left(\int_{0}^{1} f(w) \mathrm{d} x\right)^{2}}=\lambda F(w), \quad 0<x<1, \quad w(0)=0,
$$

where $F(\cdot)=f(\cdot) /\left(\int_{0}^{1} f(\cdot) \mathrm{d} x\right)^{2}$ and $\lambda$ is a positive parameter (eigenvalue). The related linearized eigenvalue problem of (2.1) for a function $\phi=\phi(x ; \lambda) \in \mathbb{R}$ ( $\phi$ is actually assumed to be a real-valued function) is then

$$
\phi^{\prime}-\lambda \delta F(w ; \phi)=-\rho \phi, \quad 0<x<1, \quad \phi(0)=\phi_{0}=0,
$$

where $\phi=\phi(x ; \lambda), \rho=\rho(w, \lambda)(\rho, \phi)$ is the eigenpair and $\delta F(w ; \phi)$ is the first variation (or Gâteaux derivative) of $F$ at $w$ in the direction of $\phi(F(w ; \phi):=F(w+\epsilon \phi)=J(\epsilon)$ and $\left.\delta F(w ; \phi)=J^{\prime}(0)=\lim _{\epsilon \rightarrow 0}((F(w+\epsilon \phi)-F(w)) / \epsilon)\right)$.

As regards the first variation $\delta F(w ; \phi)$, we have

$$
\delta F(w ; \phi)=\frac{f^{\prime}(w) \phi}{\left(\int_{0}^{1} f(w) \mathrm{d} x\right)^{2}}-\frac{2 f(w) \int_{0}^{1} f^{\prime}(w) \phi \mathrm{d} x}{\left(\int_{0}^{1} f(w) \mathrm{d} x\right)^{3}} .
$$

Below, in order to simplify the expressions, we use the shorthand notation

$$
I_{\nu k}(w, \phi):=\int_{0}^{1} f^{(\nu)}(w(x)) \phi^{k}(x) \mathrm{d} x,
$$

and $I_{\nu}(w):=I_{\nu 0}(w, \phi), \nu, k=0,1,2,3, \ldots, f^{(\nu)}(w)=\left(\mathrm{d}^{\nu} / \mathrm{d} w^{\nu}\right) f(w)$, thus

$$
\delta F(w ; \phi)=\frac{f^{\prime}(w) \phi}{I_{0}^{2}(w)}-\frac{2 f(w) I_{11}(w, \phi)}{I_{0}^{3}(w)} .
$$

We now have the following lemma concerning the eigenpair of problem (2.2). 
Lemma 2.1. Problem (2.2) has the eigenpair $(\rho, \phi)$, where $\phi(x)>0, x \in(0,1]$, and its spectrum is a continuum of eigenvalues in $\mathbb{R}$, generated by $\rho=\rho(w, \lambda)$ for each $\lambda \in\left(0, \lambda^{*}\right]$. The eigenvalue $\rho=\rho(w, \lambda)$ is continuous with respect to $\lambda$.

Proof. First, if $\phi(x)$ is a real-valued function, then equation (2.2) implies that $\rho \in \mathbb{R}$. We now write equation (2.2) in a different way:

$$
\phi^{\prime}+(g(x)+\rho) \phi=q h(x),
$$

where $q=-I_{11}(w, \phi)$ is a number,

$$
h(x)=h(x ; w(x))=\lambda \frac{2 f(w(x))}{I_{0}^{3}(w)}>0
$$

and

$$
g(x)=g(x ; w(x))=-\lambda \frac{f^{\prime}(w(x))}{I_{0}^{2}(w)} .
$$

Problem (2.2) can be written under the equivalent integral formulation:

$$
\phi(x)=q\left[\exp \left(-\int_{0}^{x} g(z) \mathrm{d} z-\rho x\right)\right] \int_{0}^{x} h(s)\left[\exp \left(\int_{0}^{s} g(z) \mathrm{d} z+\rho s\right)\right] \mathrm{d} s .
$$

The above form of $\phi$ implies that if a non-trivial $\phi$ satisfying (2.2) exists, then it is positive ( $\phi$ actually does not change sign in $(0,1)$ and can be taken to be positive).

Now we can normalize $\phi$ so that $q=1$. Therefore, there exists a real-valued function $\phi$ satisfying (2.2) if we can find $\rho \neq 0$ satisfying the following equation:

$$
1=-I_{11}(w, \phi)=\int_{0}^{1}-f^{\prime}(w(x)) \phi(x) \mathrm{d} x, \quad \text { where } \phi(x)=\phi(x ; \rho) .
$$

This can be written as

$$
1=\int_{0}^{1}-f^{\prime}(w(x))\left[\int_{0}^{x} h(x-s) \exp [-\rho s-G(x)+G(x-s)] \mathrm{d} s\right] \mathrm{d} x
$$

for $G(s)=\int_{0}^{s} g(z) \mathrm{d} z$. Therefore,

$$
1=\int_{0}^{1} \exp (-\rho s)\left[\int_{s}^{1}\left(-f^{\prime}(w(x)) h(x-s) \exp [-G(x)+G(x-s)]\right) \mathrm{d} x\right] \mathrm{d} s
$$

or

$$
1=\int_{0}^{1} Y(s) \exp (-\rho s) \mathrm{d} s
$$

where $Y(s)=Y(s ; w)>0$ for $-f^{\prime}(w) h(x-s) \exp [-G(x)+G(x-s)]>0$ and $0<s<$ $x<1$ (the eigenvalue $\rho$ is a real number). For a fixed $\lambda$, the $w$ are also fixed. Now, for each $Y(s)>1$ we have only one real solution $\rho>0$, which satisfies equation (2.3). Also, for each $Y(s)<1$ we have only one real solution $\rho<0$ and for $Y(s)=1$ we have only the trivial solution $\rho=0$. The eigenvalues $\rho=\rho(w, \lambda) \in C\left(\left(0, \lambda^{*}\right] ; \mathbb{R}\right)$ since $w(x ; \lambda)$, $g(x)=g(x ; \lambda)$ and $Y(s)=Y(s ; w)$ are continuous functions with respect to $\lambda \in\left(0, \lambda^{*}\right]$. This proves the lemma. 
It is known that the spectrum for problem (2.1) can be either an interval closed from the right or an open one. Here we consider the case where the spectrum for problem $(2.1)$ is a closed interval from the right and that there exists a unique turning point $\left(\lambda^{*},\left\|w^{*}\right\|_{\infty}\right)$ with $\left\|w^{*}\right\|_{\infty}=w^{*}(1)=M^{*}<\infty$ (see Figure $1 c$ ); at $\lambda<\lambda^{*}$ two steady states correspond to $w_{1}, w_{2}$ with $w_{1}<w_{2}$, while at $\lambda=\lambda^{*}$ they correspond to $w_{1}=w_{2}=w^{*}$. We need the following lemma.

Lemma 2.2. Let $w_{1}, w_{2}$ with $w_{1}<w_{2}$ be the solutions of (2.1) at $\lambda<\lambda^{*}$. Then $\rho_{1}=\rho\left(w_{1}, \lambda\right) \leqslant 0, \rho_{2}=\rho\left(w_{2}, \lambda\right) \geqslant 0$ and $\rho^{*}=\rho\left(w^{*}, \lambda^{*}\right)=0$, where $\rho$ represents the eigenvalues of problem (2.2).

Proof. We assume that $u(x, t)=w(x)+\epsilon \phi(x) \mathrm{e}^{\rho t}+O\left(\epsilon^{2}\right)$, with $\phi(x)>0$, for $x \in(0,1]$ and for some $\epsilon \in \mathbb{R}$. Then we find, to the first order in $\epsilon$, that $\phi$ and $\rho$ must satisfy problem $(2.2)$. We also know that $w_{1}$ is asymptotically stable, $w_{2}$ is unstable, and $w^{*}$ is stable from below and unstable from above (see $[\mathbf{2 2}]$ ). These imply that $\rho_{1} \leqslant 0, \rho_{2} \geqslant 0$ and $\rho^{*}=0$.

Thus, the lower branch of the response diagram (Figure 1c) is asymptotically stable with $\rho_{1}=\rho\left(w_{1}, \lambda\right) \leqslant 0$ ( $\rho$ is the eigenvalue of (2.2) for $\lambda<\lambda^{*}$ at $w$ ), while the upper branch is unstable with $\rho_{2}=\rho\left(w_{2}, \lambda\right) \geqslant 0$ (see also $[\mathbf{1 1}, \mathbf{1 9}, \mathbf{2 0}, \mathbf{2 3}]$ ). This continues to hold (with a suitable understanding of the 'lower and upper branch') even if there are more turning points $P^{*}$. Moreover, from Lemma 2.2 we have $\rho^{*}=\rho\left(w^{*}, \lambda^{*}\right)=0$, where $\rho$ is the eigenvalue of linearized problem (2.2) (for related results, see also $[\mathbf{2}, \mathbf{1 1}, \mathbf{2 3}]$ ). Because of Lemmas 2.1 and 2.2, problem $(2.2)$ at $\lambda=\lambda^{*}$, with $\phi^{*}(x)>0$, becomes

$$
\phi^{* \prime}-\lambda^{*} \delta F\left(w^{*} ; \phi^{*}\right)=0, \quad 0<x<1, \quad \phi^{*}(0)=\phi_{0}^{*}=0 .
$$

Now, in order to find an upper bound for $t^{*}$, we take the difference,

$$
v=v(x, t)=v(x, t ; \lambda)=u(x, t ; \lambda)-w^{*}(x)=u-w^{*} .
$$

Since $w^{*}$ is bounded, $v$ blows up at the same time as $u$, and in the same manner, i.e. globally. Hence $t^{*}=t^{*}(u)=t^{*}(v)\left(t^{*}(u)\right.$ is the blow-up time for $\left.u\right)$ and $v(x, t) \rightarrow \infty$ as $t \rightarrow t^{*}-$ for all $x \in(0,1]$. In what follows, we find an $A$-problem (see below $\left.(2.13)\right)$, where $A=A(t)$ blows up and is such that $A(t) \leqslant c\|v(\cdot, t)\|_{\infty}$ where $c=1 / \sup _{x} \phi^{*}(x)$ as long as $v(x, t) \geqslant \psi(x, t)=A(t) \phi^{*}(x)$. The latter relation and (2.5) imply that $t^{*}(u)=$ $t^{*}(v) \leqslant T^{*}=T^{*}(A)$, for some $T^{*}$. Thus, we find an upper bound $T^{*}$ for $t^{*}(u)$.

Therefore, we obtain

$$
\begin{aligned}
v_{t}=u_{t} & =-u_{x}+\lambda F(u)=-u_{x}+\lambda F(u)-\lambda^{*} F\left(w^{*}\right)+w^{* \prime} \\
& =-v_{x}+\left(\lambda-\lambda^{*}\right) F(u)+\lambda^{*}\left(F(u)-F\left(w^{*}\right)\right) .
\end{aligned}
$$

By writing $J(\epsilon)=F\left(w^{*}+\epsilon v\right), 0 \leqslant \epsilon \leqslant 1$, whence $J(0)=F\left(w^{*}\right)$ and $J(1)=F(u)$, Taylor's formula gives

$$
F(u)-F\left(w^{*}\right)=J(1)-J(0)=J^{\prime}(0)+\frac{1}{2} J^{\prime \prime}(\xi),
$$


for some $\xi=\xi(t) \in(0,1)$, where

$$
J^{\prime}(0)=\delta F\left(w^{*} ; v\right)=\left[\frac{\mathrm{d}}{\mathrm{d} \epsilon} J(\epsilon)\right]_{\epsilon=0} .
$$

Also,

$$
\delta^{2} F(z ; v)=\frac{f^{\prime \prime}(z) v^{2}}{I_{0}^{2}(z)}-\frac{4 v f^{\prime}(z) I_{11}(z, v)}{I_{0}^{3}(z)}-\frac{2 f(z) I_{22}(z, v)}{I_{0}^{3}(z)}+\frac{6 f(z) I_{11}^{2}(z, v)}{I_{0}^{4}(z)},
$$

where $z=w^{*}+\xi v$ and $\delta^{2} F(z ; v)=J^{\prime \prime}(\xi)$ is the second Gâteaux derivative. Thus, from equation (2.6) we get the problem

$$
\begin{gathered}
v_{t}+v_{x}=\left(\lambda-\lambda^{*}\right) F(u)+\lambda^{*} \delta F\left(w^{*} ; v\right)+\frac{1}{2} \lambda^{*} J^{\prime \prime}(\xi), \quad 0<x<1, \quad t>t_{1}, \\
v(0, t)=0, \quad t>t_{1}, \\
v\left(x, t_{1}\right)=u\left(x, t_{1}\right)-w^{*}(x) \geqslant 0, \quad 0 \leqslant x \leqslant 1
\end{gathered}
$$

(it is easily seen, due to (1.4), that there exists a $t_{1} \in\left(0, t^{*}\right)$ such that $v(x, t)=u(x, t)-$ $w^{*}(x)>0$ in $(0,1]$ for every $\left.t>t_{1}\right)$.

Now we set $v=u-w^{*}=\theta \hat{v}$, for $0<\theta=\lambda-\lambda^{*} \ll 1$. Thus, problem (2.7) becomes

$$
\begin{gathered}
\theta \hat{v}_{t}+\theta \hat{v}_{x}=\theta F(u)+\lambda^{*} \theta \delta F\left(w^{*} ; \hat{v}\right)+\frac{1}{2} \lambda^{*} J^{\prime \prime}(\xi), \quad 0<x<1, \quad t>t_{1}, \\
\hat{v}(0, t)=0, \quad t>t_{1}, \\
\theta \hat{v}\left(x, t_{1}\right)=u\left(x, t_{1}\right)-w^{*}(x) \geqslant 0, \quad 0<x<1 .
\end{gathered}
$$

This is simplified to

$$
\hat{v}_{t}+\hat{v}_{x}=F(u)+\lambda^{*} \delta F\left(w^{*} ; \hat{v}\right)+\frac{1}{2} \lambda^{*} \theta \hat{J}^{\prime \prime}(\xi), \quad 0<x<1, \quad t>t_{1},
$$

where $J(\xi)=\theta^{2} \hat{J}(\xi)=\theta^{2} \delta^{2} F(z ; \hat{v})$. Now we find a lower solution $\psi$ for $\hat{v}$-problem (2.8). Therefore, we require that $\psi=\psi(x, t)$ satisfies

$$
\psi_{t}+\psi_{x} \leqslant F(u)+\lambda^{*} \delta F\left(w^{*} ; \psi\right)+\frac{1}{2} \lambda^{*} \theta \delta^{2} F(z ; \psi) .
$$

Setting $\psi(x, t)=A(t) \phi^{*}(x)$ and $\dot{A}(t)=(\mathrm{d} / \mathrm{d} t) A(t)$, we obtain

$$
\dot{A}(t) \phi^{*}+A(t) \phi^{* \prime}-\lambda^{*} A(t) \delta F\left(w^{*} ; \phi^{*}\right) \leqslant F(u)+\frac{1}{2} \lambda^{*} \theta \delta^{2} F(z ; \psi) .
$$

Using $\phi^{*}$-problem (2.4), equation (2.10) becomes

$$
\dot{A}(t) \phi^{*} \leqslant F(u)+\frac{1}{2} \lambda^{*} \theta \delta^{2} F(z ; \psi) .
$$

Owing to relation (1.4) and the fact that $u$ blows up as $t \rightarrow t^{*}-$, we see that there exists a $\beta$ such that $F(u)>\beta A(t) \phi^{*}(x)>0$, for some $t \in\left[t^{*}-\varepsilon, T^{*}(A)\right)$, where $\varepsilon>0$ and $T^{*}=T^{*}(A) \geqslant t^{*}$ is the maximum time of existence (the blow-up time) of $A(t)$. Therefore, it is sufficient to consider, for $t>\tau_{1}=\max \left\{t_{1}, t^{*}-\varepsilon\right\}$,

$$
\dot{A}(t) \phi^{*}(x) \leqslant \beta A(t) \phi^{*}(x)+\frac{1}{2} \lambda^{*} \theta A^{2}(t) \delta^{2} F\left(z ; \phi^{*}\right) .
$$


For $0<\theta \ll 1$, i.e. $\lambda$ close to $\lambda^{*}$, we can find $\beta_{1}>0$, so that we get

$$
\dot{A}(t) \phi^{*} \leqslant \beta_{1} A(t) \phi^{*} \leqslant \beta A(t) \phi^{*}+\frac{1}{2} \lambda^{*} \theta A^{2}(t) \delta^{2} F\left(z ; \phi^{*}\right), \quad t>\tau_{1} .
$$

Taking $c$ small enough so that $\theta \leqslant c / A(t)$ (for some fixed $\theta$ we choose $c$, and $c_{1}$ (see below), so that $\theta \leqslant c / A(t)$, where $c$ is approximately the time that $u$ is smaller than order one, i.e. $u(x, t)$ is bounded, $A(\tau) \phi^{*} \theta+w^{*} \leqslant u(x, t)$ and $\left.0<t^{*}-\tau \ll 1\right)$ we have that $A(t) \leqslant c_{1} \mathrm{e}^{\beta_{1} t} \leqslant c / \theta$ with $c_{1}=A\left(\tau_{1}\right) \mathrm{e}^{-\beta_{1} \tau_{1}}$ and this holds for time

$$
t=\tau=\frac{1}{\beta_{1}} \ln \left(\frac{c}{\theta c_{1}}\right)
$$

We can now obtain an upper estimate $T_{u}^{*}$ for $t^{*}(u), T_{u}^{*}=\tau+t_{1}^{*}>t^{*}(u)=t^{*}$, where $t_{1}^{*}$ is the blow-up time of the problem:

$$
\begin{gathered}
u_{t}(x, t)+u_{x}(x, t)=\lambda \frac{f(u(x, t))}{\left(\int_{0}^{1} f(u(x, t)) \mathrm{d} x\right)^{2}}, \quad 0<x<1, \quad t>\tau, \\
u(0, t)=0, \quad t>\tau, \quad u(x, \tau)=w^{*}+c \phi^{*} \geqslant 0, \quad 0<x<1,
\end{gathered}
$$

and $t_{1}^{*} \ll \tau$.

\subsection{A lower bound for $t^{*}$}

We take $u_{0}(x)$ such that $u_{0}(x)<w^{*}(x)$ for $0<x<1$ and $u_{0}(0)=w^{*}(0)=0$. Let $u^{*}=u^{*}(x, t)=u\left(x, t ; \lambda^{*}\right)$ be the solution to (1.1) with $u_{0}^{*}=u_{0}$.

In the following we use a similar concept to those in $[\mathbf{1 7}, \mathbf{1 8}]$. Therefore, we set $u=$ $u^{*}+u_{1}$ and we will prove that $u \leqslant u^{*}+\psi_{1}=w^{*}-\hat{u}+\psi_{1} \leqslant w^{*}-\psi+\psi_{1}$, where $\hat{u}$ is given by $\hat{u}=w^{*}-u^{*}>0$ and satisfies (2.14), $u_{1}$ solves (2.23) (see below), $\psi_{1}$ is an upper solution to the $u_{1}$-problem, and $\psi$ is a lower solution to the $\hat{u}$-problem, i.e. $\psi_{1} \geqslant u_{1}$ and $\psi \leqslant \hat{u}$. The $\hat{u}$-problem is defined by

$$
\begin{array}{rlrl}
\hat{u}_{t} & =-u_{t}^{*}=u_{x}^{*}-\lambda^{*} F\left(u^{*}\right)-w^{* \prime}+\lambda^{*} F\left(w^{*}\right) & \\
& =-\hat{u}_{x}-\lambda^{*}\left(F\left(u^{*}\right)-F\left(w^{*}\right)\right), & & 0<x<1, \quad 0<t<T, \\
\hat{u}(0, t) & =w^{*}(0)-u^{*}(0, t)=0, & & 0<t<T, \\
\hat{u}(x, 0) & =\hat{u}_{0}(x)=w^{*}(x)-u_{0}^{*}(x), & & 0<x<1,
\end{array}
$$

with $\hat{u}_{0}>0$, hence $\hat{u}>0$, for $0<x<1,0<t<T<t^{*}$ and for some $T>0$. We write $J(\epsilon)=F\left(w^{*}-\epsilon \hat{u}\right), 0 \leqslant \epsilon \leqslant 1$ and examine the difference,

$$
F\left(u^{*}\right)-F\left(w^{*}\right)=J(1)-J(0)=J^{\prime}(0)+\frac{1}{2} J^{\prime \prime}(\xi), \quad 0<\xi<1,
$$

where

$$
J^{\prime}(0)=\delta F\left(w^{*} ;-\hat{u}\right)=-\delta F\left(w^{*} ; \hat{u}\right)=\lambda^{*} \frac{f^{\prime}\left(w^{*}\right) \hat{u}}{I_{0}^{2}\left(w^{*}\right)}-\frac{2 \lambda^{*} f\left(w^{*}\right) I_{11}\left(w^{*}, \hat{u}\right)}{I_{0}^{3}\left(w^{*}\right)}
$$


and

$$
\begin{aligned}
J^{\prime \prime}(\xi) & =\delta^{2} F(z ;-\hat{u})=\delta^{2} F(z ; \hat{u}) \\
& =\frac{f^{\prime \prime}(z) \hat{u}^{2}}{I_{0}^{2}(z)}-\frac{4 \hat{u} f^{\prime}(z) I_{11}(z, \hat{u})}{I_{0}^{3}(z)}-\frac{2 f(z) I_{22}(z, \hat{u})}{I_{0}^{3}(z)}+\frac{6 f(z) I_{11}^{2}(z, \hat{u})}{I_{0}^{4}(z)},
\end{aligned}
$$

with $0<z=w^{*}-\xi \hat{u}<w^{*}$, for some $\xi=\xi(t) \in(0,1)$.

Thus, equations $(2.14 a)$ and $(2.15)$ give

$$
L(\hat{u}):=\hat{u}_{t}+\hat{u}_{x}-\lambda^{*} \delta F\left(w^{*} ; \hat{u}\right)+\frac{1}{2} \lambda^{*} \delta^{2} F(z ; \hat{u})=0 .
$$

Now we introduce the function

$$
\psi=\psi(x, t)=\frac{c \phi^{*}(x)}{t+t_{0}}+\frac{u_{2}(x)}{\left(t+t_{0}\right)^{2}}
$$

where $c, t_{0}$ (positive constants), $u_{2}=u_{2}(x) \geqslant 0$ are to be determined and $\phi^{*}=\phi^{*}(x)$ satisfies problem (2.4). For

$$
u^{*}=w^{*}-\psi-r=w^{*}-\frac{c \phi^{*}(x)}{t+t_{0}}-\frac{u_{2}(x)}{\left(t+t_{0}\right)^{2}}-r \geqslant 0,
$$

where

$$
r=r(x, t)=\frac{u_{3}(x)}{\left(t+t_{0}\right)^{3}}+\frac{u_{4}(x)}{\left(t+t_{0}\right)^{4}}+\cdots \quad(r \in \mathbb{R}),
$$

since $u^{*} \rightarrow w^{*}-$ or $(\psi+r) \rightarrow 0+$ as $t \rightarrow \infty\left(u_{0}^{*}(x)<w^{*}(x)\right)$, actually it holds: $u^{*}<w^{*}$ and $\psi+r>0$ for all $t \geqslant 0[\mathbf{2 2}]$. Thus, we have

$$
u_{t}^{*}+u_{x}^{*}=\lambda^{*} F\left(u^{*}\right)
$$

or

$$
\begin{aligned}
& \frac{c \phi^{*}}{\left(t+t_{0}\right)^{2}}+w^{* \prime}-\frac{c \phi^{* \prime}}{t+t_{0}}-\frac{u_{2}^{\prime}}{\left(t+t_{0}\right)^{2}}+\cdots \\
& \quad=\lambda^{*} F\left(w^{*}-\frac{c \phi^{*}}{t+t_{0}}-\frac{u_{2}}{\left(t+t_{0}\right)^{2}}+\cdots\right) \\
& \quad=\lambda^{*} F\left(w^{*}\right)-\lambda^{*} \frac{c}{t+t_{0}} \delta F\left(w^{*} ; \phi^{*}\right)-\frac{\lambda^{*}}{\left(t+t_{0}\right)^{2}}\left[\delta F\left(w^{*} ; u_{2}\right)-\frac{1}{2} c^{2} \delta^{2} F\left(w^{*} ; \phi^{*}\right)\right]+\cdots
\end{aligned}
$$

Equating terms of the same order with respect to powers of $1 /\left(t+t_{0}\right)$ and taking into account the $\phi^{*}$-problem, we have

$$
\left(c \phi^{*}-u_{2}^{\prime}\right)=-\frac{1}{2} \lambda^{*} c^{2} \delta^{2} F\left(w^{*} ; \phi^{*}\right)-\lambda^{*} \delta F\left(w^{*} ; u_{2}\right) .
$$

We choose $c$ such that

$$
c \int_{0}^{1} \phi^{*} \mathrm{~d} x=c^{2} \frac{1}{2} \lambda^{*}\left|\int_{0}^{1} \delta^{2} F\left(w^{*} ; \phi^{*}\right) \mathrm{d} x\right|,
$$


which implies that $c=2 / \lambda^{*}\left|\int_{0}^{1} \delta^{2} F\left(w^{*} ; \phi^{*}\right) \mathrm{d} x\right|>0$, since $c=0$ is rejected and provided that $\int_{0}^{1} \phi^{*} \mathrm{~d} x=1$ (it should be $c>0$, otherwise we would have $u^{*}>w^{*}$ for $t \gg 1$ ).

Then we need to estimate $u_{2}$ by the inequality

$$
u_{2}^{\prime} \leqslant \lambda^{*} \delta F\left(w^{*} ; u_{2}\right)+c \phi^{*}+\frac{1}{2} \lambda^{*} c^{2} \delta^{2} F\left(z ; \phi^{*}\right),
$$

where $0<z=w^{*}-\xi \hat{u}<w^{*}$, for some $\xi=\xi(t) \in(0,1)$.

Therefore, by taking $m_{0}=\frac{1}{2} \lambda^{*} c^{2} \inf _{x}\left\{\delta^{2} F\left(z ; \phi^{*}\right)\right\}$ we have

$$
u_{2}^{\prime} \leqslant \lambda^{*}\left[\frac{f^{\prime}\left(w^{*}\right) u_{2}}{I_{0}^{2}\left(w^{*}\right)}-\frac{2 f\left(w^{*}\right) I_{11}\left(w^{*}, u_{2}\right)}{I_{0}^{3}\left(w^{*}\right)}\right]+c \phi^{*}+m_{0} .
$$

Now taking

$$
c_{1}=\inf _{x}\left\{\lambda^{*} \frac{f^{\prime}\left(w^{*}\right)}{I_{0}^{2}\left(w^{*}\right)}\right\}, \quad c_{2}=\sup _{x}\left\{-\frac{2 f\left(w^{*}\right)}{I_{0}^{3}\left(w^{*}\right)}\right\}
$$

and $q=\int_{0}^{1} f^{\prime}\left(w^{*}\right) u_{2} \mathrm{~d} x=I_{11}\left(w^{*}, u_{2}\right)<0$, we need only to estimate $u_{2}$ by the expression

$$
u_{2}^{\prime}(x) \leqslant c_{1} u_{2}(x)+c_{2} q+c \phi^{*}(x)+m_{0} .
$$

This implies that we need

$$
u_{2}^{\prime}(x) \leqslant c_{1} u_{2}(x)+c_{2} q+m_{1},
$$

where $m_{1}=c \inf _{x} \phi^{*}(x)+m_{0}=m_{0}$; hence,

$$
u_{2} \leqslant \frac{c_{2} q+m_{1}}{c_{1}}\left(\mathrm{e}^{c_{1} x}-1\right)
$$

for $u_{2}(0)=0$, which is satisfied if

$$
q=\frac{c_{2} q+m_{1}}{c_{1}} \int_{0}^{1} f^{\prime}\left(w^{*}\right)\left(\mathrm{e}^{c_{1} x}-1\right) \mathrm{d} x .
$$

Therefore,

$$
q=\frac{m_{1} m_{2}}{c_{1}-c_{2} m_{2}} \quad \text { for } m_{2}=\int_{0}^{1} f^{\prime}\left(w^{*}\right)\left(\mathrm{e}^{c_{1} x}-1\right) \mathrm{d} x,
$$

which can be estimated.

Now, substituting $\psi$, which is known, for $\hat{u}$ in (2.16) and taking into account the $\phi^{*}$ problem and (2.18), we obtain (for the operator $L$ (see (2.16)))

$$
\begin{aligned}
L(\psi)= & \frac{c}{t+t_{0}}\left(\phi^{* \prime}-\lambda^{*} \delta F\left(w^{*} ; \phi^{*}\right)\right) \\
& \quad+\frac{1}{\left(t+t_{0}\right)^{2}}\left(u_{2}^{\prime}-\lambda^{*} \delta F\left(w^{*} ; u_{2}\right)-c \phi^{*}+\frac{1}{2} \lambda^{*} c^{2} \delta^{2} F\left(z ; \phi^{*}\right)\right)+O\left(\frac{1}{\left(t+t_{0}\right)^{3}}\right) \\
\leqslant & 0 .
\end{aligned}
$$

The last inequality holds since the term of order $1 /\left(t+t_{0}\right)^{2}$ dominates the term of order $1 /\left(t+t_{0}\right)^{3}$, this due to the $u^{*}$-problem, actually since $u^{*} \rightarrow w^{*}-$ or $(\psi+r) \rightarrow 0+$ as $t \rightarrow \infty$ and that $\psi+r>0$ for all $t>0$. 
Requiring $\psi(x, 0) \leqslant \hat{u}_{0}$ and now knowing $u_{2}$, since $\hat{u}_{0}=w^{*}-u_{0}^{*}=w^{*}-u_{0}$, we choose

$$
t_{0}=\sup _{x} \frac{c \phi^{*}+\sqrt{c^{2} \phi^{*^{2}}+4 \hat{u}_{0} u_{2}}}{2 \hat{u}_{0}}=\sup _{x} \frac{E(x)}{2 \hat{u}_{0}}<\infty,
$$

in $[0,1]$. Provided that $u_{0}^{*}(x)=u_{0}(x)=w^{*}(x)-\hat{u}_{0}(x)$ is chosen so that $E(x)=O\left(\hat{u}_{0}(x)\right)$ as $x \rightarrow 0+$, without loss of generality we may choose $u_{0} \rightarrow 0+$ appropriately as $x \rightarrow 0+$. Then we have that $\psi$ is a lower solution to the $\hat{u}$-problem and thus $\psi \leqslant \hat{u}$.

We now write $u=u^{*}+u_{1} \leqslant w^{*}$ and find an upper solution to the $u_{1}$-problem. The equation for $u_{1}$ is

$$
\begin{array}{r}
u_{1 t}=-u_{1 x}+\left(\lambda-\lambda^{*}\right) F\left(w^{*}\right)+\lambda\left(F(u)-F\left(w^{*}\right)\right)-\lambda^{*}\left(F\left(u^{*}\right)-F\left(w^{*}\right)\right), \\
0<x<1, \quad t>0 .
\end{array}
$$

We again examine the difference $\lambda\left(F(u)-F\left(w^{*}\right)\right)$ and write $v=u-w^{*}\left(-w^{*}<v<0\right)$, $J_{1}(\epsilon)=F\left(w^{*}+\epsilon v\right), 0 \leqslant \epsilon \leqslant 1$. We have

$$
\begin{aligned}
\lambda\left(F(u)-F\left(w^{*}\right)\right) & =\lambda\left(J_{1}(1)-J_{1}(0)\right) \\
& =\lambda\left(\frac{f^{\prime}\left(w^{*}\right) v}{I_{0}^{2}\left(w^{*}\right)}-\frac{2 f\left(w^{*}\right) I_{11}\left(w^{*} ; v\right)}{I_{0}^{3}\left(w^{*}\right)}\right)+\frac{1}{2} \lambda J_{1}^{\prime \prime}\left(\xi_{1}\right) \\
& =\lambda^{*} \delta F\left(w^{*} ; v\right)+\left(\lambda-\lambda^{*}\right) \delta F\left(w^{*} ; v\right)+\frac{1}{2} \lambda \delta^{2} F(z ; v) \\
& =\lambda^{*} \delta F\left(w^{*} ; v\right)+Q\left(w^{*}, z, v\right),
\end{aligned}
$$

where

$$
Q\left(w^{*}, z, v\right)=\left(\lambda-\lambda^{*}\right) \delta F\left(w^{*} ; v\right)+\frac{1}{2} \lambda \delta^{2} F(z ; v)
$$

and

$$
\begin{aligned}
J_{1}^{\prime \prime}\left(\xi_{1}\right)=\delta^{2} F(z ; v)=\frac{1}{I_{0}^{4}(z)}\left[I_{0}^{2}(z) v^{2} f^{\prime \prime}(z)\right. & -4 v f^{\prime}(z) I_{11}(z, v) I_{0}(z) \\
& \left.-2 f(z) I_{0}(z) I_{22}(z, v)+6 f(z) I_{0}(z) I_{11}^{2}(z, v)\right],
\end{aligned}
$$

$z=w^{*}+\xi_{1} v, \xi_{1}=\xi_{1}(t) \in(0,1)$.

Also, setting $u^{*}=w^{*}-\hat{u}$ and $J_{2}(\epsilon)=F\left(w^{*}-\epsilon \hat{u}\right), 0 \leqslant \epsilon \leqslant 1$, we may have for the quantity $\lambda^{*}\left(F\left(u^{*}\right)-F\left(w^{*}\right)\right)$ :

$$
\begin{aligned}
-\lambda^{*}\left(F\left(u^{*}\right)-F\left(w^{*}\right)\right) & =-\lambda^{*}\left(J_{2}(1)-J_{2}(0)\right) \\
& =\lambda^{*}\left(\frac{f^{\prime}\left(w^{*}\right) \hat{u}}{I_{0}^{2}\left(w^{*}\right)}-\frac{2 f\left(w^{*}\right) I_{11}\left(w^{*}, \hat{u}\right)}{I_{0}^{3}\left(w^{*}\right)}\right)-\frac{1}{2} \lambda^{*} J_{2}^{\prime \prime}\left(\xi_{2}\right) \\
& =\lambda^{*} \delta F\left(w^{*} ; \hat{u}\right)-\frac{1}{2} \lambda^{*} \delta^{2} F(\zeta ; \hat{u}),
\end{aligned}
$$

with

$$
\begin{aligned}
J_{2}^{\prime \prime}\left(\xi_{2}\right)=\delta^{2} F(\zeta ; \hat{u})=\frac{1}{I_{0}^{4}(\zeta)}\left[I_{0}^{2}(\zeta) \hat{u}^{2} f^{\prime \prime}(\zeta)\right. & -4 \hat{u} f^{\prime}(\zeta) I_{11}(\zeta, \hat{u}) I_{0}(\zeta) \\
& \left.-2 f(\zeta) I_{0}(\zeta) I_{22}(\zeta, \hat{u})+6 f(\zeta) I_{0}(\zeta) I_{11}^{2}(\zeta, \hat{u})\right],
\end{aligned}
$$


where $\zeta=w^{*}-\xi_{2} \hat{u}, \xi_{2}=\xi_{2}(t) \in(0,1)$.

The $u_{1}$-problem (2.20), with relations $(2.21),(2.22)$, now becomes

$$
\begin{gathered}
u_{1 t}=-u_{1 x}+\left(\lambda-\lambda^{*}\right) F\left(w^{*}\right)+\lambda^{*} \delta F\left(w^{*} ; v\right)+Q\left(w^{*}, z, v\right) \\
+\lambda^{*} \delta F\left(w^{*} ; \hat{u}\right)-\frac{1}{2} \lambda^{*} \delta^{2} F(\zeta ; \hat{u}), \quad 0<x<1, \quad t>0, \\
u_{1}(0, t)=0, \quad t>0, \\
u_{1}(x, 0)=u_{0}(x)-u_{0}^{*}(x)=0, \quad 0<x<1,
\end{gathered}
$$

where $0<z<w^{*}, 0<\zeta<w^{*}, 0<\hat{u}<w^{*}, u<u_{1}<w^{*}$ as long as $u<w^{*}$, so that $Q\left(w^{*}, z, v\right), J_{2}^{\prime \prime}\left(\xi_{2}\right)$ are bounded from above and below.

Hence, for a fixed $\lambda>\lambda^{*}$, there exist some constants $B_{1}, B_{2}$ and $B>0$ such that

$$
Q\left(w^{*}, z, v\right)<B_{1}<\frac{1}{2}\left(\lambda-\lambda^{*}\right) B, \quad-\frac{1}{2} \lambda^{*} J_{2}^{\prime \prime}\left(\xi_{2}\right)<B_{2}<\frac{1}{2}\left(\lambda-\lambda^{*}\right) B
$$

and $Q-\frac{1}{2} \lambda^{*} J_{2}^{\prime \prime}<\left(\lambda-\lambda^{*}\right) B$. From (2.20)-(2.23) we obtain

$$
\begin{aligned}
u_{1 t} \leqslant-u_{1 x}+\left(\lambda-\lambda^{*}\right) & F\left(w^{*}\right)+\lambda^{*}\left[\frac{f^{\prime}\left(w^{*}\right) v}{I_{0}^{2}\left(w^{*}\right)}-\frac{2 f\left(w^{*}\right) I_{11}\left(w^{*}, v\right)}{I_{0}^{3}\left(w^{*}\right)}\right] \\
& +\lambda^{*}\left[\frac{f^{\prime}\left(w^{*}\right) \hat{u}}{I_{0}^{2}\left(w^{*}\right)}-\frac{2 f\left(w^{*}\right) I_{11}\left(w^{*}, \hat{u}\right)}{I_{0}^{3}\left(w^{*}\right)}\right]+\left(\lambda-\lambda^{*}\right) B .
\end{aligned}
$$

Owing to the fact that $u_{1}=u-u^{*}=u-w^{*}+w^{*}-u^{*}=v+\hat{u}$, the previous relation becomes

$$
u_{1 t} \leqslant-u_{1 x}+\left(\lambda-\lambda^{*}\right) F\left(w^{*}\right)+\lambda^{*}\left[\frac{f^{\prime}\left(w^{*}\right) u_{1}}{I_{0}^{2}\left(w^{*}\right)}-\frac{2 f\left(w^{*}\right) I_{11}\left(w^{*}, u_{1}\right)}{I_{0}^{3}\left(w^{*}\right)}\right]+\left(\lambda-\lambda^{*}\right) B .
$$

Now we introduce $\psi_{1}(x, t)=\left[\left(\lambda-\lambda^{*}\right) \Lambda\left(t+t_{0}\right)\right] \phi^{*}(x)$, where $\Lambda$ is a constant which is determined so that $\psi_{1}$ will be an upper solution to the $u_{1}$-problem. Here $\phi^{*}$ again satisfies problem (2.4).

By substituting $-\psi_{1}$ for $u_{1}$ on the right-hand side of the above relation, we get

$$
\begin{gathered}
-\psi_{1 x}+\left(\lambda-\lambda^{*}\right) F\left(w^{*}\right)+\lambda^{*}\left[\frac{f^{\prime}\left(w^{*}\right) \psi_{1}}{I_{0}^{2}\left(w^{*}\right)}-\frac{2 f\left(w^{*}\right) I_{11}\left(w^{*}, \psi_{1}\right)}{I_{0}^{3}\left(w^{*}\right)}\right]+\left(\lambda-\lambda^{*}\right) B \\
=\left(\lambda-\lambda^{*}\right) F\left(w^{*}\right)+\left(\lambda-\lambda^{*}\right) B \leqslant\left(\lambda-\lambda^{*}\right) \Lambda \phi^{*}=\frac{\partial \psi_{1}}{\partial t}, \\
F\left(w^{*}(x)\right)+B \leqslant \Lambda \phi^{*}(x), \quad \text { or } \sup _{x} F\left(w^{*}(x)\right)+B \leqslant \Lambda \phi^{*}(x) .
\end{gathered}
$$

Since

$$
\sup _{x} F\left(w^{*}(x)\right)=\frac{f(0)}{f^{2}\left(w^{*}(1)\right)}
$$

it is sufficient to take

$$
\frac{f(0)}{f^{2}\left(w^{*}(1)\right)}+B=\Gamma \leqslant \Lambda \phi^{*}(x) \quad \text { or } \quad 0<\Gamma \leqslant \Lambda \inf _{x \in[\gamma, 1]} \phi^{*}(x)=\Lambda \Theta,
$$


for some $\gamma \in(0,1)$. Also $\psi_{1}(x, 0)=\left[\left(\lambda-\lambda^{*}\right) \Lambda t_{0}\right] \phi^{*}(x)>u_{1}(x, 0)=0$, and

$$
\psi_{1}(\gamma, t)=\left[\left(\lambda-\lambda^{*}\right) \Lambda\left(t+t_{0}\right)\right] \phi^{*}(\gamma)>u_{1}(\gamma, t) \geqslant 0
$$

Relations (2.25), (2.26) lead us to choose

$$
\Lambda=\max \left\{\frac{w^{*}(\gamma)}{\left(\lambda-\lambda^{*}\right) t_{0} \phi^{*}(\gamma)}, \frac{\Gamma}{\Theta}\right\}
$$

for some $\gamma>0$ (such a $\gamma$ exists since $\phi^{*}(0)=0$ and $\phi^{*}(x)>0$ in $\left.(0,1]\right)$. Then, $\psi_{1}$ is a 'restricted' upper solution for the $u_{1}$-problem in the interval $[\gamma, 1][\mathbf{2 1}]$. Here we should note that $u$ blows up globally (see relation $(1.4 b)$ ). This means that $u(x, t)$ is bounded for $(x, t) \in[0, \gamma] \times[0, T]$ for some $T<t^{*}$. In other words, $u$ is bounded in $[\gamma, 1] \times[0, T]$ (actually, here we require $u<w^{*}$ (see below)) and hence a lower bound for $t^{*}$ can be found by working in the restricted interval $[\gamma, 1]$.

Hence, $u \leqslant w^{*}$ as long as $\psi-\psi_{1} \geqslant 0$. Thus, we have

$$
u=u_{1}+u^{*} \leqslant w^{*}-\frac{c \phi^{*}}{t+t_{0}}-\frac{u_{2}}{\left(t+t_{0}\right)^{2}}+\left(\lambda-\lambda^{*}\right) \Lambda\left(t+t_{0}\right) \phi^{*} .
$$

The right-hand side of the above relation is no greater than $w^{*}$, as long as $\psi \geqslant \psi_{1}$ or

$$
\frac{c \phi^{*}}{\left(t+t_{0}\right)}+\frac{u_{2}}{\left(t+t_{0}\right)^{2}} \geqslant\left(\lambda-\lambda^{*}\right) \Lambda \phi^{*}\left(t+t_{0}\right), \quad x \in[\gamma, 1]
$$

Hence, $u \leqslant w^{*}$ as long as $\psi-\psi_{1} \geqslant 0$; it is sufficient that $u \leqslant w^{*}$ for any $x \in[\gamma, 1]$, for some $\gamma>0$, due to the fact that $u$ blows up globally.

Therefore, for simplicity, since $0<\lambda-\lambda^{*} \ll 1$, it is sufficient to choose

$$
\frac{c \phi^{*}}{t+t_{0}} \geqslant\left(\lambda-\lambda^{*}\right) \Lambda \phi^{*}\left(t+t_{0}\right), \quad x \in[\gamma, 1]
$$

so that

$$
c \geqslant\left(\lambda-\lambda^{*}\right) \Lambda\left(t+t_{0}\right)^{2} .
$$

Thus, we obtain

$$
t \leqslant c^{1 / 2} \Lambda^{-1 / 2}\left(\lambda-\lambda^{*}\right)^{-1 / 2}-t_{0}
$$

which, for $\lambda$ sufficiently close to $\lambda^{*}\left(\lambda>\lambda^{*}\right)$, gives

$$
t \lesssim c^{1 / 2} \Lambda^{-1 / 2}\left(\lambda-\lambda^{*}\right)^{-1 / 2}=t_{l}\left(\lambda-\lambda^{*}\right)^{-1 / 2}
$$

Hence, as long as $u=u(x, t)<w^{*}(x), x \in[\gamma, 1]$, at $t=t_{l}\left(\lambda-\lambda^{*}\right)^{-1 / 2}$, we deduce that $t^{*}>t_{l}\left(\lambda-\lambda^{*}\right)^{-1 / 2}$, i.e. $t_{l}\left(\lambda-\lambda^{*}\right)^{-1 / 2}$ is a lower bound for $t^{*}$ with $t_{l}=c^{1 / 2} \Lambda^{-1 / 2}$. 


\section{Asymptotic estimate of $t^{*}$ for small $\lambda-\lambda^{*}>0$}

We now examine the special case $f(s)=\mathrm{e}^{-s}$. Motivated by $\S 2$, we wish to find an estimate for the blow-up time $t^{*}$ in problem (1.1) as an asymptotic series of $\eta=\theta^{1 / 2}=$ $\left(\lambda-\lambda^{*}\right)^{1 / 2} \ll 1, \eta>0$. We again assume that $u_{0}(x)<w^{*}(x)$ for $0<x \leqslant 1$, with $u_{0}(0)=w^{*}(0)=0$.

Following concepts similar to those in $[\mathbf{1 7}, \mathbf{1 8}]$, and prompted by numerical calculations, we consider three intervals of time, say I, II and III.

In I and III, $t$ varies by $O(1)$ as $\theta=\left(\lambda-\lambda^{*}\right) \rightarrow 0$ and we expand $u \sim u^{*}+\theta v_{1}+\theta^{2} v_{2}+\cdots$ as $\theta \rightarrow 0+$, where $u^{*}$ satisfies problem (1.1) at $\lambda=\lambda^{*}$. More precisely, in I we have $u^{*}<w^{*}$ since $u_{0}=u_{0}^{*}<w^{*}$ in $(0,1]$, while in III we have $u^{*}>w^{*}$. Moreover, in I,

$$
u^{*}(x, t) \sim w^{*}(x)-\frac{2 \phi^{*}(x)}{\lambda^{*} J_{2} t} \quad \text { as } t \rightarrow \infty,
$$

with $J_{2}=\int_{0}^{1} R_{2}\left(w^{*}, \phi^{*}\right) \mathrm{d} x$ and $R_{2}\left(w^{*}, \phi^{*}\right)=\delta^{2} F\left(w^{*} ; \phi^{*}\right)$, where $R_{2}$ follows from the second term of Taylor's expansion (see $\S 2$ or below relation (3.1)). This can be obtained by assuming that $u^{*} \sim w^{*}+(K / t) \phi^{*}$ as $t \rightarrow \infty, u^{*}<w^{*}$, and, substituting this expansion in the equation for $u^{*}$, we find that $K=-2 / \lambda^{*} J_{2}$, provided that $\int_{0}^{1} \phi^{*}(x) \mathrm{d} x=1$.

In III $\left(u^{*}>w^{*}\right)$, we again expand $u$ in a similar manner as in I, but now

$$
u^{*}(x, t) \sim w^{*}(x)-\frac{2 \phi^{*}(x)}{\lambda^{*} J_{2} \hat{t}}
$$

as $\hat{t} \rightarrow-\infty$ and $t=t_{*}+\hat{t}>0$ for some large $t_{*}\left(t_{*} \sim t^{*}<\infty\right.$ for $\left.\theta \rightarrow 0\right)$ and $u^{*}$ becomes infinite at some finite time $\hat{t}_{*}$ as $\theta \rightarrow 0$.

In II, we expand $u \sim w^{*}+\eta v_{1}+\eta^{2} v_{2}+\cdots$ as $\eta \rightarrow 0$ and, on making a change in timescale $t=\tau / \eta$, equation (1.1) gives

$$
\eta^{2} v_{1 \tau}+\eta^{3} v_{2 \tau}+\cdots+w_{x}^{*}+\eta v_{1 x}+\eta^{2} v_{2 x}+\cdots=\lambda R(\eta) \quad \text { as } \eta \rightarrow 0
$$

where

$$
F(u) \sim \hat{R}(x, t ; \eta):=R(\eta)=\frac{\mathrm{e}^{-\left(w^{*}+\eta v_{1}+\eta^{2} v_{2}+\cdots\right)}}{\left(\int_{0}^{1} \mathrm{e}^{-\left(w^{*}+\eta v_{1}+\eta^{2} v_{2}+\cdots\right)}\right)^{2} \mathrm{~d} x} \quad \text { as } \eta \rightarrow 0 .
$$

We require an expansion for $R(\eta)$ as follows:

$$
R(\eta)=R(0)+\eta R^{\prime}(0)+\frac{1}{2} \eta^{2} R^{\prime \prime}(0)+\cdots
$$

From (3.1), (3.2) we obtain

$\eta^{2} v_{1 \tau}+\eta^{3} v_{2 \tau}+\cdots+w_{x}^{*}+\eta v_{1 x}+\eta^{2} v_{2 x}+\cdots=\left(\lambda^{*}+\eta^{2}\right)\left(R(0)+\eta R^{\prime}(0)+\frac{1}{2} \eta^{2} R^{\prime \prime}(0)+\cdots\right)$.

As regards the boundary condition $u(0, t)=0$ at $x=0$, we have

$$
w^{*}(0)+\eta v_{1}(0, \tau)+\eta^{2} v_{2}(0, \tau)+\cdots=0 .
$$


We equate the terms of zero order $\left(O(1)\right.$ or $\left.O\left(\eta^{0}\right)\right)$ and obtain

$$
w^{* \prime}=\lambda^{*} R(0), \quad 0<x<1, \quad w^{*}(0)=0,
$$

where $R(0)=\mathrm{e}^{-w^{*}} /\left(\int_{0}^{1} \mathrm{e}^{-w^{*}} \mathrm{~d} x\right)^{2}$. Problem (3.3) is actually problem (1.3). By looking now at the terms of $O(\eta)$ we have

$$
v_{1 x}(x, \tau)=\lambda^{*} R^{\prime}(0), \quad 0<x<1, \quad \tau>0, \quad v_{1}(0, \tau)=0, \quad \tau>0,
$$

where

$$
R^{\prime}(0)=\delta F\left(w^{*} ; v_{1}\right)=-\frac{\mathrm{e}^{-w^{*}} v_{1}}{\left(\int_{0}^{1} \mathrm{e}^{-w^{*}} \mathrm{~d} x\right)^{2}}+\frac{2 \mathrm{e}^{-w^{*}} \int_{0}^{1} \mathrm{e}^{-w^{*}} v_{1} \mathrm{~d} x}{\left(\int_{0}^{1} \mathrm{e}^{-w^{*}} \mathrm{~d} x\right)^{3}}
$$

Problem (3.4) has the form of problem (2.4), thus we can write

$$
v_{1}(x, \tau)=a(\tau) \phi^{*}(x),
$$

where now we normalize $\phi^{*}$ according to $\int_{0}^{1} \phi^{* 2}(x) \mathrm{d} x=1$ and we denote the normalized $\phi^{*}$ again by $\phi^{*}$. Looking next at the $O\left(\eta^{2}\right)$ terms we have

$$
v_{1 \tau}+v_{2 x}=R(0)+\frac{1}{2} \lambda^{*} R^{\prime \prime}(0)
$$

which becomes

$$
\begin{aligned}
v_{1 \tau}+v_{2 x}=\frac{\mathrm{e}^{-w^{*}}}{S_{0}^{2}\left(\phi^{*}\right)}+\frac{2 \lambda^{*} \mathrm{e}^{-w^{*}} v_{1} S_{1}\left(v_{2}\right)}{S_{0}^{3}\left(\phi^{*}\right)} & +\frac{2 \lambda^{*} \mathrm{e}^{-w^{*}} S_{1}\left(v_{2}\right)}{S_{0}^{3}\left(\phi^{*}\right)} \\
& -\frac{\lambda^{*} \mathrm{e}^{-w^{*}} S_{2}\left(v_{2}\right)}{S_{0}^{3}\left(\phi^{*}\right)}+\frac{3 \lambda^{*} \mathrm{e}^{-w^{*}} S_{1}^{2}\left(v_{2}\right)}{S_{0}^{4}\left(\phi^{*}\right)},
\end{aligned}
$$

where now we define $I_{\nu k}\left(w^{*}, v\right)=(-1)^{\nu} S_{k}(v)$ with $S_{k}(v)=\int_{0}^{1} \mathrm{e}^{-w^{*}} v^{k} \mathrm{~d} x, k=0,1,2,3$, and $S_{k}\left(\phi^{*}\right)=S_{k}$.

Multiplying (3.6) by $\phi^{*}$, integrating over [0,1], using (3.5) and the normalization of $\phi^{*}$, we obtain

$$
\begin{aligned}
\dot{a}(\tau)=\int_{0}^{1}\left(-\phi^{* \prime}-\frac{\lambda^{*} \mathrm{e}^{-w^{*}} \phi^{*}}{S_{0}^{2}}+\frac{2 \lambda^{*} \mathrm{e}^{-w^{*}} S_{1}}{S_{0}^{3}}\right) & v_{2} \mathrm{~d} x+\frac{S_{1}}{S_{0}^{2}} \\
& +\lambda^{*} \frac{a^{2}(\tau)}{2 S_{0}^{4}}\left(S_{3} S_{0}^{2}-6 S_{1} S_{2} S_{0}+6 S_{1}^{3}\right) .
\end{aligned}
$$

Now we look at the equation for $\phi^{*}$, which is

$$
\phi^{* \prime}=-\frac{\lambda^{*} \mathrm{e}^{-w^{*}} \phi^{*}}{S_{0}^{2}}+\frac{2 \lambda^{*} \mathrm{e}^{-w^{*}} S_{1}}{S_{0}^{3}}, \quad 0<x<1, \quad \phi^{*}(0)=0,
$$

and integrate over $[0,1]$, to obtain

$$
\phi^{*}(1)=\frac{\lambda^{*} S_{1}}{S_{0}^{2}}
$$


Moreover, multiplying (3.6) by $\phi^{*}$ and then integrating over [0,1], we obtain

$$
\frac{\phi^{* 2}(1) S_{0}^{3}}{2 \lambda^{*}}=-S_{2} S_{0}+2 S_{1}^{2}
$$

In the same way, but multiplying by $\phi^{* 2}$, we get

$$
\frac{\phi^{* 3}(1) S_{0}^{3}}{3 \lambda^{*}}=-S_{3} S_{0}+2 S_{2} S_{1} .
$$

Now, for the quantity $S=S_{3} S_{0}^{2}-6 S_{2} S_{1} S_{0}+6 S_{1}^{3}$, after using (3.9) and relations (3.10), (3.11) we obtain

$$
S=S_{1}^{3}\left(-2+2 \frac{\lambda^{*}}{S_{0}}-\frac{\lambda^{* 2}}{3 S_{0}^{2}}\right)
$$

The quantity $S$ is positive as long as the quantity $S_{q}=-6 S_{0}^{2}+6 \lambda^{*} S_{0}-\lambda^{* 2}$ is positive. We know the relations $\lambda^{*}=M^{2} / \mu$, where $M=\max \left\{w^{*}(x)\right\}=w^{*}(1)$, for $0 \leqslant x \leqslant 1$ and $\mu=\lambda^{*} / S_{0}^{2}$. Using these relations we find that $S_{q}$ is positive provided

$$
S_{q} M^{2}=\lambda^{* 2}\left(-6+6 M-M^{2}\right)
$$

is positive. We have (see [22]) that $M \sim 1.5936$ and $\lambda^{*} \sim 0.6476$. The above quantity, for these values, is positive $\left(S_{q} M^{2} \sim 0.4286\right)$.

Therefore, equation (3.7) can be written as

$$
\dot{a}(\tau)=\frac{S_{1}}{S_{0}^{2}}+\lambda^{*} \frac{S}{2 S_{0}^{4}} a^{2}(\tau), \quad a(\tau) \rightarrow-\infty \text { as } \tau \rightarrow 0,
$$

which has as a solution

$$
a(\tau)=\left(\frac{B}{K}\right)^{1 / 2} \tan \left[\tau(B K)^{1 / 2}-\frac{1}{2} \pi\right]
$$

for $K=\lambda^{*} S / 2 S_{0}^{4}, B=S_{1} / S_{0}^{2}$ (this choice of initial condition as $\tau \rightarrow 0+$ gives constant of integration $-\frac{1}{2} \pi$ ). Returning to the original time variable, this expression becomes

$$
A(t)=\left(\frac{B}{K}\right)^{1 / 2} \tan \left[t\left(\lambda-\lambda^{*}\right)^{1 / 2}(B K)^{1 / 2}-\frac{1}{2} \pi\right] .
$$

Because $u=w^{*}+\eta v_{1}+\cdots$ and $v_{1}(x, t)=A(t) \phi^{*}(x)$, it is obvious that $u$ ceases to exist at time

$$
t^{*} \sim t_{b}=t_{u}\left(\lambda-\lambda^{*}\right)^{-1 / 2}
$$

where $t_{u}=\pi(4 B K)^{-1 / 2}$ and $t_{b}$ is the blow-up time of $a(\tau)=a\left(t\left(\lambda-\lambda^{*}\right)^{-1 / 2}\right)=A(t)$. 


\section{Numerical solutions}

We solve problem (1.1) by using a two-step upwind scheme. For the linear terms we apply the usual form of the scheme:

$$
v_{j}^{n+1}=u_{j}^{n}-r\left(u_{j}^{n}-u_{j-1}^{n}\right)+\lambda F\left(u_{j}^{n}\right),
$$

where $u_{j}^{n}$ is the temperature at the $n$th time level and at the $j$ th space grid, $r=\delta t / \delta x$, and the non-local term $F\left(u_{j}^{n}\right)$ is evaluated at the $n$th time step. For this term we have

$$
F\left(u_{j}^{n}\right)=\frac{f\left(u_{j}^{n}\right)}{\left(\int_{0}^{1} f\left(u_{j}^{n}\right) \mathrm{d} x\right)^{2}} .
$$

The integral in the denominator is evaluated by Simpson's rule. In the next step we evaluate $w$ :

$$
w_{j}^{n+1}=u_{j}^{n}-r\left(v_{j}^{n+1}-v_{j-1}^{n+1}\right)+\lambda F\left(v_{j}^{n+1}\right) .
$$

Finally, $u$ at the $(n+1)$ th time step is approximated by

$$
u_{j}^{n+1}=\frac{1}{2}\left(v_{j}^{n+1}+w_{j}^{n+1}\right) .
$$

In Figure 2 we use this scheme to solve the problem numerically for $f(u)=\mathrm{e}^{-u}$ and on taking $u(x, 0)=0$. We see that for $\lambda<\lambda^{*}$ the solution $u$ tends to a steady state, for $\lambda=\lambda^{*}$ the behaviour is similar, and for $\lambda>\lambda^{*}$ the solution blows up (the decay is faster for $\lambda<\lambda^{*}$ than it is for $\left.\lambda=\lambda^{*}\right)$. More precisely, in Figure 2 the maxima of solutions are plotted against time.

In Figure 3, we plot the numerical solution of $u$ for $\lambda=0.5476$.

\section{Upper bounds of $t^{*}$ for sufficiently large $u_{0}(x)$ and $\lambda \leqslant \lambda^{*}$}

In this section we study the case of $\lambda \leqslant \lambda^{*}$, where the steady-state problem (1.3) has two (bounded) solutions $w_{1}(x), w_{2}(x)$ with $w_{2}(x)>w_{1}(x)\left(w_{1}=w_{2}=w^{*}\right.$ at $\left.\lambda=\lambda^{*}\right)$ for $x \in(0,1]$ (see Figure 1c). Following concepts similar to those in the case of the upper bound for $t^{*}$ when $\lambda>\lambda^{*}$, we may proceed in one of two ways: either (i) as in [17] or (ii) as in $\S 2$.

\subsection{First method (as in [17])}

Again we set $v(x, t)=u(x, t)-w_{2}(x), 0 \leqslant x \leqslant 1, t>0$, with $v_{0}(x)=u_{0}(x)-w_{2}(x)>0$, $0<x \leqslant 1$ and $v_{0}(0)=u_{0}(0)-w_{2}(0)=0$. The function $w_{2}(x)$ is the greatest steadystate solution to problem (1.3), while $u(x, t)$ is the unique solution to problem (1.1) which blows up globally in finite time, and (1.4) holds. Therefore, $v(x, t) \rightarrow \infty$ and $F(u)=F\left(v+w_{2}\right) \rightarrow \infty$ as $t \rightarrow t^{*}-$.

Also,

$$
F(u)-F\left(w_{2}\right)=F\left(v+w_{2}\right)-F\left(w_{2}\right)=\delta F\left(w_{2} ; v\right)+\frac{1}{2} \delta^{2} F(s ; v) .
$$




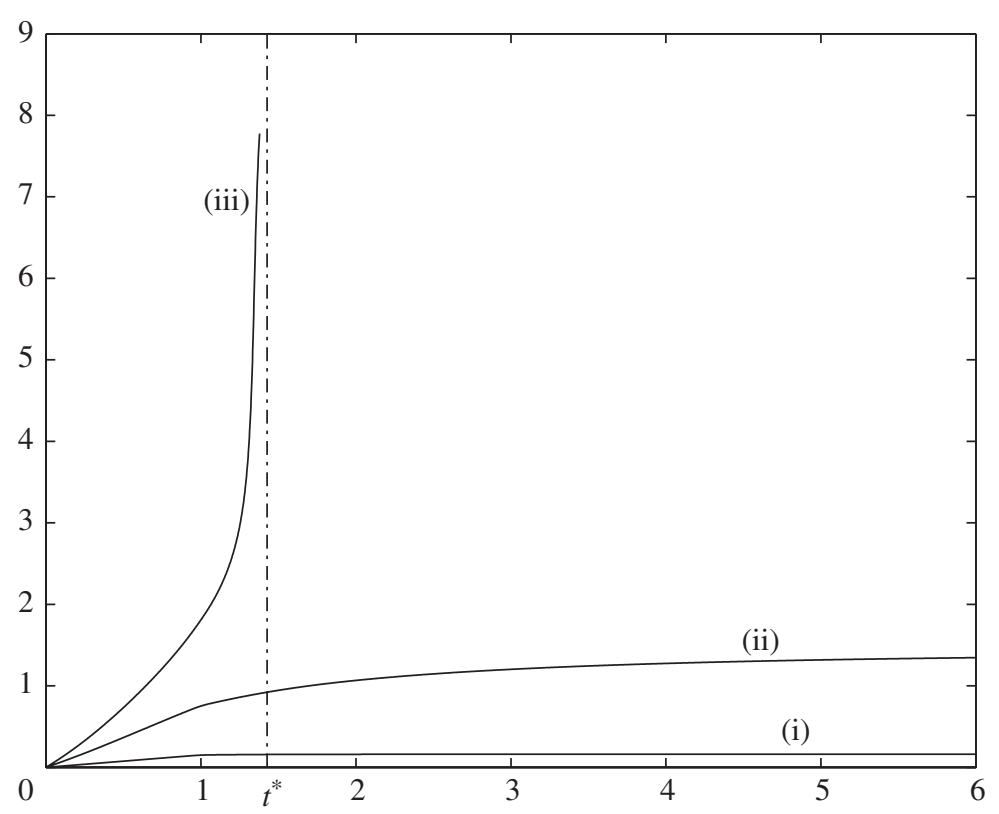

Figure 2. Numerical solution to problem (1.1). We plot $\max _{x}(u(x, t))=u(1, t)=M(t)$, for $x$ in $[0,1]$, against time for $\delta x=0.033, \delta t=0.002$ (the upper curve, (iii), corresponds to $\lambda=1.1476>\lambda^{*}=0.6476$, the intermediate curve, (ii), to $\lambda=\lambda^{*}$, and the lower one, (i), to $\left.\lambda=0.1476<\lambda^{*}\right)$. Also the dash-dotted line corresponds to the asymptotic estimate of the blow-up time $t^{*} \sim 1.3367$ for $\lambda=1.1476$. To obtain this estimate we calculate $w^{*}$ numerically by an iteration scheme and then we solve the equation for $\phi^{*}$ using the appropriate normalization.

Then $v$ satisfies the problem

$$
\begin{gathered}
v_{t}+v_{x}=\lambda \delta F\left(w_{2} ; v\right)+\frac{1}{2} \lambda \delta^{2} F(s ; v), \quad 0<x<1, \quad t>0, \\
v(0, t)=0, \quad t>0, \\
v_{0}=v(x, 0)=u(x, 0)-w_{2}(x) \geqslant 0, \quad 0 \leqslant x \leqslant 1,
\end{gathered}
$$

where $v_{0}=0$ only at $x=0, s=s(x, t)=w_{2}+\xi v, \xi=\xi(t) \in(0,1), F\left(w_{2}+\xi v\right):=L(\xi)$ and $\delta^{2} F(s, v):=L^{\prime \prime}(\xi)$. Moreover, $\delta^{2} F(s ; v) \rightarrow \infty$ as $t \rightarrow t^{*}-$, otherwise $v(x, t)$ would not blow up (in finite time).

Now we introduce the function $\Psi$, for which we need to satisfy

$$
\Psi_{t}+\Psi_{x} \leqslant \lambda \delta F\left(w_{2} ; \Psi\right)+\frac{1}{2} \lambda \delta^{2} F(z ; \Psi)=\lambda\left(F\left(w_{2}+\Psi\right)-F\left(w_{2}\right)\right),
$$

where $\Psi(x, t)=A(t) \phi(x), \phi(x)=\phi(x ; \rho), z=w_{2}+\epsilon \Psi, \epsilon=\epsilon(t) \in(0,1), J(\epsilon)=F\left(w_{2}+\right.$ $\epsilon \Psi), J^{\prime \prime}(\epsilon)=\delta^{2} F(z ; \Psi), \phi(x)$ satisfies problem (2.2) and $A(t)$ satisfies problem (5.7) (see below). We require the function $\Psi$ to be a lower solution to problem (5.1) which also blows up, due to the blow-up of $A(t)$ at $T^{*}$; since $\Psi \leqslant v$, we have $T^{*}=T^{*}(A) \geqslant t^{*}(v)=$ $t^{*}(u)=t^{*}$, and actually this blow-up is global because of the form of $\Psi$.

Setting $\Psi(x, t)=A(t) \phi(x)$ in relation (5.2) we obtain

$$
\dot{A}(t) \phi+A(t) \phi^{\prime}-\lambda \delta F\left(w_{2} ; \phi\right) A(t) \leqslant \frac{1}{2} \lambda \delta^{2} F(z ; \Psi) .
$$




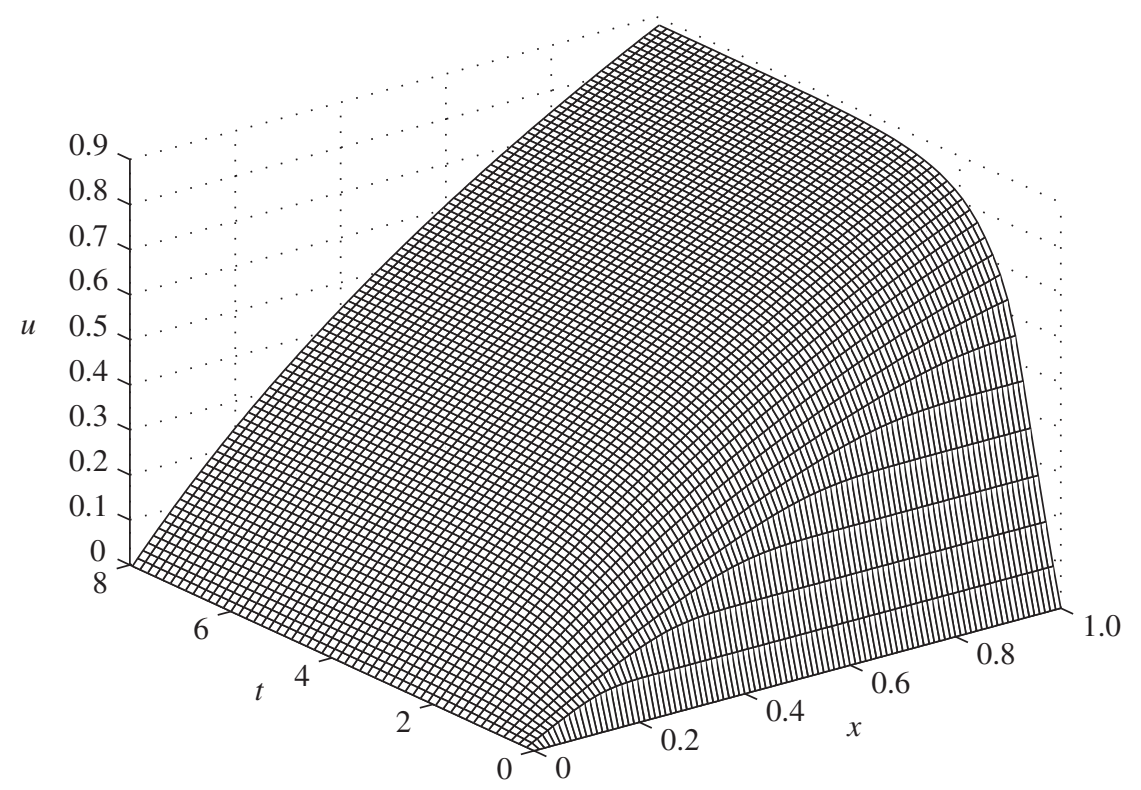

Figure 3. Numerical solution to problem (1.1) for $\lambda=0.5476<\lambda^{*}$.

Also, by the same argument as for $v$, we have that $J^{\prime \prime}(\epsilon)=\delta^{2} F(z ; \Psi) \rightarrow \infty, \Psi \rightarrow \infty$ as $t \rightarrow T^{*}-$, otherwise $\Psi(x, t)$ would not blow up. Then, for $J^{\prime \prime}(\epsilon)$, we have

$$
\begin{aligned}
J^{\prime \prime}(\epsilon) & =\Psi^{2}\left[\frac{f^{\prime \prime}(z)}{I_{0}^{2}(z)}-\frac{4 f^{\prime}(z) I_{11}(z, \Psi)}{\Psi I_{0}^{3}(z)}-\frac{2 f(z) I_{22}(z, \Psi)}{\Psi^{2} I_{0}^{3}(z)}+\frac{6 f(z) I_{11}^{2}(z, \Psi)}{\Psi^{2} I_{0}^{4}(z)}\right] \\
& =\Psi^{2}\left[\frac{f^{\prime \prime}(z)}{I_{0}^{2}(z)}-\frac{4 f^{\prime}(z) I_{11}(z, \phi) A}{A \phi I_{0}^{3}(z)}-\frac{2 f(z) I_{22}(z, \phi) A^{2}}{A^{2} \phi^{2} I_{0}^{3}(z)}+\frac{6 f(z) I_{11}^{2}(z, \phi) A^{2}}{A^{2} \phi^{2} I_{0}^{4}(z)}\right] \\
& =\Psi^{2} \Gamma(x, t) \\
& =A^{2}(t) \phi(x) \Gamma_{1}(x, t)
\end{aligned}
$$

where $\Gamma_{1}(x, t)=\Gamma(x, t) \phi(x)$. Since $\Psi$ blows up globally, we have that $F\left(w_{2}+\Psi\right)-F\left(w_{2}\right) \sim$ $F\left(w_{2}+\Psi\right) \sim F(\Psi) \rightarrow \infty$ as $t \rightarrow T^{*}-$ for every $x \in(0,1]$.

Now we impose an extra condition on the function $f$,

$$
f(s) \leqslant \frac{c}{s^{2}}, \quad c>0, \quad \text { for } s \gg 1,
$$

and we have the following lemma.

Lemma 5.1. Let $f$ satisfy (1.2) and (5.5), then $J^{\prime \prime}(\epsilon)=\delta^{2} F(z ; \Psi) \geqslant \Lambda A^{2}(t) \phi(x)>0$ for $\Psi \gg 1$, or $A \gg 1$, where $\Lambda$ is a positive constant.

Proof. Since $\Psi$ blows up globally, $\delta^{2} F(z ; \Psi) \rightarrow \infty$ as $\Psi \rightarrow \infty$. If we assume that the conclusion of the lemma does not hold, then owing to (5.3) we have $\delta^{2} F(z ; \Psi) \lesssim$ $K_{0} A^{p}(t) \phi(x)$ as $A(t) \rightarrow \infty$ or as $t \rightarrow T^{*}-$, for some $1<p<2$ and $K_{0}>0$. Otherwise $\Psi$ 
does not blow up. The latter, the fact that $F\left(w_{2}+\Psi\right)-F\left(w_{2}\right) \sim F(\Psi)$, for $\Psi \gg 1$, and (5.4) give

$$
\begin{aligned}
F\left(w_{2}+\Psi\right)-F\left(w_{2}\right) & =\delta F\left(w_{2} ; \Psi\right)+\frac{1}{2} \delta^{2} F(z ; \Psi) \\
& =\delta F\left(w_{2}, \phi\right) A(t)+\frac{1}{2} \Gamma(x, t) \Psi^{2} \\
& \sim F(\Psi) \\
& \lesssim K_{0} A^{p} \phi, \quad \text { for } A \gg 1 \text { and } 1<p<2 .
\end{aligned}
$$

Also, by using the mean-value theorem we obtain

$$
F(\Psi)=\frac{f(\Psi(x, t))}{\left(\int_{0}^{1} f(\Psi(x, t)) \mathrm{d} x\right)^{2}}=\frac{f(A(t) \phi(x))}{f^{2}(A(t) \phi(\xi))},
$$

where $\xi=\xi(t) \in(0,1)$. Then, due to the continuity of $\phi(x)$, we can always find $x_{0}=$ $x_{0}(t) \in(0,1)$ such that $0<\phi\left(x_{0}(t)\right)<\min \left\{\phi(\xi(t)), \phi^{2}(\xi(t))\right\}$. This implies that

$$
\frac{f\left(A(t) \phi\left(x_{0}(t)\right)\right)}{f(A(t) \phi(\xi(t)))}>1
$$

and, as $t \rightarrow T^{*}-$, we have

$$
F\left(\Psi\left(x_{0}, t\right)\right)=\frac{f\left(A(t) \phi\left(x_{0}\right)\right)}{f^{2}(A(t) \phi(\xi))}>\frac{1}{f(A(t) \phi(\xi))},
$$

and

$$
\frac{1}{f(A(t) \phi(\xi))}<\frac{f\left(A(t) \phi\left(x_{0}\right)\right)}{f^{2}(A(t) \phi(\xi))}=F\left(\Psi\left(x_{0}, t\right)\right) \lesssim K_{0} A^{p}(t) \phi\left(x_{0}\right) .
$$

Then, by using the previous relation and condition (5.5), we obtain

$$
K_{0} A^{p}(t) \phi\left(x_{0}(t)\right) \gtrsim \frac{1}{f(A(t) \phi(\xi(t)))} \geqslant \frac{A^{2}(t) \phi^{2}(\xi(t))}{c} \quad \text { as } t \rightarrow T^{*} .
$$

The above inequality implies that

$$
K_{0} c \gtrsim A^{2-p}(t) \frac{\phi^{2}(\xi(t))}{\phi\left(x_{0}(t)\right)}>A^{2-p}(t)
$$

for $t$ close to $T^{*}$, but $A^{2-p}(t) \rightarrow \infty$ as $t \rightarrow T^{*}$ for $1<p<2$, while $K_{0} c<\infty$, which is a contradiction.

Owing to Lemma 5.1, relation (5.4) now becomes

$$
J^{\prime \prime}(\epsilon)=\Gamma(x, t) \Psi^{2} \geqslant \Lambda A^{2}(t) \phi(x) \quad \text { as } t \rightarrow T^{*}-, \quad x \in(0,1] .
$$

From relation (5.6), it is sufficient to take

$$
\dot{A}(t) \phi+A(t)\left[\phi^{\prime}-\lambda \delta F\left(w_{2} ; \phi\right)\right] \leqslant K_{1} A^{2}(t) \phi, \quad t>0, \quad A(0)=A_{0},
$$


where $K_{1}=\frac{1}{2} \lambda \Lambda$. Now, by using problem (2.2) and Lemmas 2.2 and 5.1 we obtain

$$
\dot{A}(t)-A(t) \rho_{2} \leqslant K_{1} A^{2}(t), \quad t>0, \quad A(0)=A_{0}, \quad \rho_{2} \geqslant 0 .
$$

Thus, we can take $A(t)$ in order to satisfy

$$
\dot{A}(t)=\rho A(t)+K A^{2}(t), \quad t \in\left[0, T^{*}\right), \quad A(0)=A_{0},
$$

with $K \leqslant K_{1}$ and $\rho=\rho_{2} \geqslant 0$.

We also take

$$
\psi(x, 0)=A(0) \phi(x) \leqslant v(x, 0)=u_{0}(x)-w_{2}(x) \quad \text { or } \quad A_{0}=A(0) \leqslant \frac{u_{0}(x)-w_{2}(x)}{\phi(x)} ;
$$

hence, we choose

$$
A_{0}=\inf _{x} \frac{u_{0}(x)-w_{2}(x)}{\phi(x)}=\inf _{x} \frac{E(x)}{\phi(x)}>0
$$

(this infimum exists provided that $u_{0}(x), u_{0}^{\prime}(x)$ are bounded in $\left.[0,1]\right)$. Moreover, $\Psi(0, t)=$ $v(0, t)$. Finally, $\Psi$ is a lower solution to the $v$-problem (5.1).

Now problem (5.7) for $A(t)$ and $\rho>0$ gives

$$
A(t)=\left[-\frac{K}{\rho}+\left(A_{0}^{-1}+\frac{K}{\rho}\right) \mathrm{e}^{-\rho t}\right]^{-1}
$$

where $K$ is a constant depending on $c$ and $\lambda$, while $A_{0}$ depends on the infimum of the difference $\left(u_{0}(x)-w_{2}(x)\right) / \phi(x)$.

Then the blow-up time $T^{*}$ for $A(t)$ is

$$
T^{*}=\frac{1}{\rho} \ln \left(\frac{\rho}{K} A_{0}^{-1}+1\right), \quad \text { for } \rho>0 .
$$

While, for $\rho=0$, problem (5.7) gives $T^{*}=\left(K A_{0}\right)^{-1}$. Since $\Psi$ is a lower solution of the $v$-problem (5.1), we obtain

$$
t^{*}=t^{*}(u)=t^{*}(v) \leqslant t^{*}(\Psi)=T^{*},
$$

hence $T^{*}$ is an upper bound for $t^{*}(u)$.

\subsection{Second method (as in $\S 2)$}

This method works only if $\rho=\rho_{2}>0$ or if $\rho=\rho_{2}=0$ and $\delta^{2} F(z ; \phi)>0$. Now let $u(x, t)=u\left(x, t ; u_{0}\right)$ be the solution to problem (1.1) for $\lambda<\lambda^{*}$ and assume, for simplicity, initial data of the form $u_{0}(x)=\sigma w_{2}(x)$, with $\sigma>1$. Then $u_{0}(x)-w_{2}(x)=$ $(\sigma-1) w_{2}(x)=\zeta w_{2}(x)$, with $\zeta=\sigma-1>0$. Also we have that $u \rightarrow w_{2}+$ as $\zeta \rightarrow 0+$. Again we set $v(x, t)=u(x, t)-w_{2}(x), 0 \leqslant x \leqslant 1, t>0$, with $v_{0}(x)=u_{0}(x)-w_{2}(x)>0$, 
$0<x \leqslant 1$, and $v_{0}(0)=u_{0}(0)-w_{2}(0)=0$. We call $v=u-w_{2}=\zeta \hat{v}$, thus problem $(5.1)$ becomes

$$
\begin{gathered}
\zeta \hat{v}_{t}+\zeta \hat{v}_{x}=\lambda \zeta \delta F\left(w_{2} ; \hat{v}\right)+\frac{1}{2} \lambda J^{\prime \prime}(\xi), \quad 0<x<1, \quad t>0 \\
\hat{v}(0, t)=0, \quad t>0, \\
\hat{v}_{0}(x)=\hat{v}(x, 0)=\frac{u_{0}(x)-w_{2}(x)}{\zeta}>0, \quad 0<x<1 .
\end{gathered}
$$

This simplifies to

$$
\hat{v}_{t}+\hat{v}_{x}=\lambda \delta F\left(w_{2} ; \hat{v}\right)+\frac{1}{2} \lambda \zeta \hat{J}^{\prime \prime}(\xi), \quad 0<x<1, \quad t>0,
$$

where $J(\xi)=\zeta^{2} \hat{J}(\xi)=\zeta^{2} \delta^{2} F(z ; \hat{v})$. Now we find a lower solution $\psi$ for the $\hat{v}$-problem. Therefore, we require $\psi=\psi(x, t)$ to satisfy

$$
\psi_{t}+\psi_{x} \leqslant \lambda \delta F\left(w_{2} ; \psi\right)+\frac{1}{2} \lambda \zeta \delta^{2} F(z ; \psi)
$$

Setting $\psi=A(t) \phi(x)$ and $\dot{A}(t)=(\mathrm{d} / \mathrm{d} t) A(t)$, we obtain

$$
\dot{A}(t) \phi+A(t)\left(\phi^{\prime}-\lambda \delta F\left(w_{2} ; \phi\right)\right) \leqslant \frac{1}{2} \lambda \zeta \delta^{2} F(z ; \psi) .
$$

Using the $\phi$-problem (2.2) and Lemma 2.2, equation (5.10) becomes

$$
\dot{A}(t) \phi-\rho_{2} \phi A(t) \leqslant \frac{1}{2} \lambda \zeta \delta^{2} F(z ; \psi), \quad \rho_{2} \geqslant 0 .
$$

Thus, we have, on setting $\rho_{2}=\rho$,

$$
\dot{A}(t) \phi(x) \leqslant \rho A(t) \phi(x)+\frac{1}{2} \lambda \zeta A^{2}(t) \delta^{2} F(z ; \phi), \quad 0<x<1, \quad t>0 .
$$

For $0<\zeta \ll 1$, in both cases, i.e. either $\rho=\rho_{2}>0$, or $\rho=\rho_{2}=0$ and $\delta^{2} F(z ; \phi)>0$, we can find $\beta>0$, so that we get (for some $\tau_{1}$ close to $T^{*}$ and $\tau_{1}<T^{*}$ )

$$
\dot{A}(t) \phi \leqslant \beta A(t) \phi \leqslant \rho A(t) \phi+\frac{1}{2} \lambda \zeta A^{2}(t) \delta^{2} F(z ; \phi), \quad t>\tau_{1}>0 .
$$

Now, taking $c$ small enough so that $\zeta \leqslant c / A(t)$ ( $c$ is approximately the time that $u$ becomes smaller than order one) we have that $A(t) \leqslant c_{1} \mathrm{e}^{\beta t} \leqslant c / \zeta$ with $c_{1}=A\left(\tau_{1}\right) \mathrm{e}^{-\beta \tau_{1}}$ and this holds for time

$$
t=\tau=\frac{1}{\beta} \ln \left(\frac{c}{\zeta c_{1}}\right)
$$

Now we can obtain an upper estimate $T_{u}^{*}$ for $t^{*}(u)$ which is $T_{u}^{*}=\tau+t_{1}^{*}>t^{*}=t^{*}(u)$, where $t_{1}^{*}$ is the blow-up time of the problem

$$
\begin{gathered}
u_{t}(x, t)+u_{x}(x, t)=\lambda \frac{f(u(x, t))}{\left(\int_{0}^{1} f(u(x, t)) \mathrm{d} x\right)^{2}}, \quad 0<x<1, \quad t>\tau, \\
u(0, t)=0, \quad t>\tau, \quad u(x, \tau)=w_{2}(x)+c \phi(x) \geqslant 0, \quad 0<x<1,
\end{gathered}
$$

and $t_{1}^{*} \ll \tau$. 


\section{Discussion}

In the present work, we estimate the blow-up time $t^{*}$ of the solution to problem (1.1). It is useful, from the point of view of applications, to know the time at which the temperature $u$ becomes infinity. In our model, this is the time that the food is burnt. Similar estimates are also known for local (the reaction-diffusion problem) as well as for non-local (the ohmic heating problem) problems $[\mathbf{1 7}, \mathbf{1 8}]$. Here the results are obtained for the case where there exists a steady-state solution $w^{*}=w\left(x ; \lambda^{*}\right)$ at $\lambda=\lambda^{*}$, either for $0<\lambda-\lambda^{*} \ll 1$, with non-negative initial data, or for $0<\lambda \leqslant \lambda^{*}$ with initial data greater than the greatest steady-state solution. The methods applied are comparison and asymptotic techniques, as well as numerical computations. Although the asymptotic estimate of $t^{*}$ is found for $f(s)=\mathrm{e}^{s}$, it is possible to estimate it for a general function $f$ satisfying (1.2).

Our main estimates, for given $\lambda, \lambda^{*}$ and $0<\lambda-\lambda^{*} \ll 1$, are upper bound $\epsilon+c_{1} \ln \left[c_{2}(\lambda-\right.$ $\left.\lambda^{*}\right)^{-1}$, lower bound $c_{3}\left(\lambda-\lambda^{*}\right)^{-1 / 2}$, and asymptotic estimate $t^{*} \sim c_{4}\left(\lambda-\lambda^{*}\right)^{-1 / 2}$ as $\lambda \rightarrow \lambda^{*}+$. For $0<\lambda \leqslant \lambda^{*}$ and given initial data $u_{0}(x)$ greater than the greatest steadystate solution $w_{2}(x)$, we have the upper estimates $c_{5} \ln \left(c_{6} A_{0}^{-1}+1\right)$ or $\epsilon+c_{7} \ln \left(c_{8} \zeta^{-1}\right)$, where $A_{0}, \zeta$ are measures of the difference $u_{0}-w_{2}$ (if $u_{0} \rightarrow w_{2}+$, then $A_{0}, \zeta \rightarrow 0+$ ), the $c_{i}$ are constants, and $0<\epsilon \ll 1$. Some numerical results are also represented in $\S 4$.

It still remains an open question how to estimate $t^{*}$ when there is no regular solution $w^{*}$ at $\lambda=\lambda^{*}$.

Acknowledgements. The authors thank Professor A. A. Lacey for several fruitful discussions. We also thank A. Delis for fruitful discussion regarding the numerical scheme.

This research was supported partly by Thalis grant 65/132400, NTUA, and by Pythagoras 68831, from the Greek Ministry of Education (the project is co-funded by the European Social Fund (75\%) and national resources (25\%): EPEAEK II-Pythagoras).

\section{References}

1. W. Allegretto And H. Xie, Existence of solutions for the time-dependent thermistor equations, IMA J. Appl. Math. 48 (1992), 271-281.

2. H. Amann, Fixed point equations and nonlinear eigenvalue problems in ordered Banach Spaces, SIAM Rev. 18 (1976), 620-709.

3. J. W. BEBERNES AND D. EBERLY, Mathematical problems from combustion theory (Springer, 1989).

4. H. Bellout, A criterion for blow-up of solutions to semilinear heat equations, SIAM J. Math. Analysis 18(3) (1987), 722-727.

5. C. H. Biss, S. A. Coombers And P. J. Skudder, The development and applications of ohmic heating for continuous processing of particulate foodstuffs, in Process engineering in the food industry (ed. R. W. Field and J.A Howell), pp. 17-27 (Elsevier, 1989).

6. J. A. CARRILlo, On a non-local elliptic equation with decreasing nonlinearity arising in plasma physics and heat conduction, Nonlin. Analysis 32 (1998), 97-115.

7. N. CHAFEE, The electric ballast resistor: homogeneous and nonhomogeneous equilibria in Nonlinear differential equations: invariance stability and bifurcations (ed. P. de Mottoni and L. Salvatori), pp. 97-127 (Academic, 1981).

8. X. Chen And A. Friedman, The thermistor problem for conductivity which vanishes at large temperature, Q. Appl. Math. 51 (1993), 101-115. 
9. G. Cimatтi, Remark on existence and uniqueness for the thermistor problem under mixed boundary conditions, Q. Appl. Math. 47 (1989), 117-121.

10. G. Cimatti, The stationary thermistor problem with a current limiting device, Proc. $R$. Soc. Edinb. A 116 (1990), 79-84.

11. M. G. CRAndall AND P. H. Rabinowitz, Bifurcation, perturbation of simple eigenvalues, and linearized stability, Arch. Ration. Mech. Analysis 582 (1973), 161-180.

12. A. A. P. De Alwis And P. J. Fryer, Operability of the ohmic heating process: electrical conductivity effects, J. Food Engng 15 (1992), 21-48.

13. A. C. Fowler, I. FrigaArd AND S. D. Howison, Temperature surges in currentlimiting circuit devices, SIAM J. Appl. Math. 52 (1992), 998-1011.

14. P. FREITAS AND M. GRINFELD, Stationary solutions of an equation modelling ohmic heating, Appl. Math. Lett. 7(3) (1994), 1-6.

15. P. J. Fryer, A. A. P. De Alwis, E. Koury, A. G. F. Stapley and L. Zhang, Ohmic processing of solid-liquid mixtures: heat generation and convection effects, J. Food Engng 18 (1993), 101-125.

16. V. A. Galaktionov and J. L. VAzQuez, The problem of blow-up in nonlinear parabolic equations, in Proc. Summer Course on Partial Differential Equations, Temuco, Chile, 1999 (Pitman, London, 2000).

17. N. I. Kavallaris, C. V. Nikolopoulos and D. E. Tzanetis, Estimates of blow-up time for a non-local problem modelling an ohmic heating process, Eur. J. Appl. Math. 13 (2002), 337-351.

18. A. A. LACEY, Mathematical analysis of thermal runaway for spartially inhomogeneous reactions, SIAM J. Appl. Math. 43 (1983), 1350-1366.

19. A. A. LACEY, Thermal runaway in a non-local problem modelling ohmic heating, I, Model derivation and some special cases, Eur. J. Appl. Math. 6 (1995), 127-144.

20. A. A. LACEY, Thermal runaway in a non-local problem modelling ohmic heating, II, General proof of blow-up and asymptotics of runaway, Eur. J. Appl. Math. 6 (1995), 201-224.

21. A. A. Lacey And D. Tzanetis, Global existence and convergence to a singular steady state for a semilinear heat equation, Proc. R. Soc. Edinb. A 105 (1986), 289-305.

22. A. A. Lacey, D. E. Tzanetis and P. M. Vlamos, Behaviour of a nonlocal reactive convective problem modelling ohmic heating of foods, Q. J. Mech. Appl. Math. 5(4) (1999), 623-644.

23. J. Lopez-Gomez, On the structure and stability of the set of solutions of a non-local problem modelling ohmic heating, J. Dynam. Diff. Eqns 10(4) (1998), 537-559.

24. C. P. Please, D. W. Schwendeman and P. S. Hagan, Ohmic heating of foods during aseptic processing, IMA J. Math. Appl. Bus. Indust. 5 (1994), 283-301.

25. D. H. SATtinger, Monotone methods in nonlinear elliptic and parabolic boundary value problems, Indiana Univ. Math. J. 21 (1972), 979-1000.

26. P. Skudder And S. BISS, Aseptic processing of food products using ohmic heating, Chem. Engr 2 (1987), 26-28.

27. R. Stirling, Ohmic heating - a new process for the food industry, Power Engng J. 6 (1987), 365-371.

28. D. E. Tzanetis, Blow-up of radially symmetric solutions of a non-local problem modelling ohmic heating, Electron. J. Diff. Eqns 2002(11) (2002), 1-26.

29. L. ZHANG AND P. J. FRYER, Models for the electrical heating of solid-liquid food mixtures, Chem. Engng Sci. 48 (1993), 633-642. 